\title{
An Experimental Investigation of the Scratch Behaviour of Polymers: 2. Influence of Hard or Soft Fillers
}

\author{
Pinar Kurkcu, Luca Andena*, Andrea Pavan \\ Dipartimento di Chimica, Materiali e Ingegneria Chimica “Giulio Natta”, Politecnico di Milano
}

\begin{abstract}
Variation in the scratch behaviour of polymers with incorporation of different types and amounts of fillers was investigated both in terms of scratch visibility and scratch hardness. Scratch tests were carried out by a microscratch tester. The effect of fillers on the scratch deformation mode and hence on scratch visibility was evaluated by examining recorded penetration depth and acoustic emission traces together with optical microscope images of the groove left on the surface. Scratch hardness was determined by using a recently proposed scratch model. Adequacy of the model to interpret the local phenomenon induced by addition of fillers was questioned.

It was demonstrated that incorporation of hard fillers can enhance the scratch hardness of polymers as well as their basic mechanical properties but leads to an increase in scratch visibility due to a change in scratch deformation mode from ductile ploughing to a brittle failure with crack formation. On the contrary, addition of soft fillers causes a reduction in scratch hardness but it also reduces scratch visibility since it improves the recovery characteristics of the polymer.
\end{abstract}

\section{Keywords}

Polymer-matrix composite, scratch testing, hardness, hard/soft fillers

\section{Introduction}

Polymers are more and more frequently being utilised in applications demanding a high surface quality such as in the automotive industry, data storage and optical products. Surface quality may be related to distinct characteristics depending on the specific application. From a mechanical point of view, one of the crucial features is the material resistance to scratch. The presence of scratches has a considerable effect on the performance of most polymer components. As a matter of fact they are not just aesthetically undesirable: they may impair the component's functionality or even its structural integrity. Hence, understanding scratch behaviour of polymers [1-6] is essential to both maintain good appearance and enhance durability.

Scratch behaviour of polymers depends on a variety of factors, which can be classified into two primary groups: (i) indenter geometry [7-9] and testing conditions, such as normal load [8,10-11], temperature [12-13] and sliding tip velocity $[8,11,13]$ and (ii) material characteristics such as elastic modulus [14-16], yield stress [15], ductility [17-18], crystallinity [7,19], hardness [16,20], surface roughness [9], surface tension [21-22]. Incorporation of fillers of different type, amount and size, is expected to have an effect on the scratch behaviour through the variation in the fundamental material properties. Thus hard particles are expected to strengthen the polymer matrix and therefore enhance the loadbearing capacity of the composite. On the contrary, soft particles are expected to decrease friction, but also reduce the material's strength [23].

The influence of fillers on the scratch behaviour of polymers has been investigated in various studies by evaluating different aspects: scratch deformation mode [24-29], scratch visibility 
[30-31], residual scratch tracks, i.e. scratch depth, width, etc. [25, 28-30] and scratch hardness $[24,28,32]$. Nevertheless, it still remains to be properly understood whether incorporation of fillers modifies the material's scratch performance directly or it simply acts by modifying the material's bulk mechanical properties.

The aim of this study is thus to investigate how the presence of hard or soft fillers affects both the scratch performance and the mechanical properties (elastic modulus and yield stress) of filled polymers. Applicability of a recent scratch model to interpret the scratch behaviour of polymer composites, in which the local phenomenon has a significant influence, is questioned.

\section{Experimental}

\subsection{Materials}

Several polymer composite materials were prepared, combining two different matrixes (a thermosetting epoxy and a thermoplastic styrene-acrylonitrile copolymer) with varying contents of either soft or hard filler. In this preliminary study, the fillers used had different sizes, depending on their availability.

\subsubsection{Preparation of Epoxy-based Composites}

The epoxy matrix used in this study was prepared from a bisphenol-A based epoxy prepolymer (Ampreg 26) and an amine-based curing agent (Ampreg Fast Hardener), supplied by SP Systems.

Two batches of glass spheres with an average diameter of $100 \mu \mathrm{m}$ and $25 \mu \mathrm{m}$ were used as hard fillers. The glass spheres were added to the epoxy pre-polymer and dispersed by gentle (to avoid entrapping air bubbles) but prolonged mechanical stirring. After that, the curing agent was slowly added and stirring was continued for a while to prevent the precipitation of glass spheres due to the density difference between glass and epoxy. Optical micrographs of sections of these glass/epoxy composites, after solidification, were taken to verify that a homogeneous dispersion of particles had been achieved; an example is shown in Fig. 1 for four different cases of particle size and content.

A commercial toughening agent (Fortegra 100, Dow Chemical) was used as a soft filler. The liquid compound was added to the epoxy pre-polymer and dispersed under the action of a magnetic stirrer rotating at a very low speed to minimize air bubble formation. Then, the curing agent was slowly added while stirring was continued for a few minutes to ensure good mixing with the epoxy pre-polymer.

The admixtures of epoxy and curing agent with either hard or soft filler were cast into a silicone mould and cured for 24 hours at $23^{\circ} \mathrm{C}$. Then, post-curing was carried out at $100^{\circ} \mathrm{C}$ for $1 \mathrm{hr}$; afterwards the samples were left to cool down to room temperature naturally by switching the oven off.

\subsubsection{Preparation of SAN-based Composites}

Styrene-acrylonitrile copolymer (SAN) and acrylonitrile-butadiene-styrene terpolymer (ABS) with varying butadiene-rubber contents were supplied by ANIC S.p.A. in powder form.

SAN/glass spheres composites were prepared by using a Brabender internal mixer. The operating temperature, speed and mixing time were $165^{\circ} \mathrm{C}, 25 \mathrm{rpm}$ and 5 minutes, respectively. Glass spheres with an average diameter of $25 \mu \mathrm{m}$ were used for the SAN-based composites. Samples of neat SAN and ABS were also treated in the Brabender under the same conditions as the SAN/glass spheres composite samples so that all SAN-based materials underwent the same thermal history. 


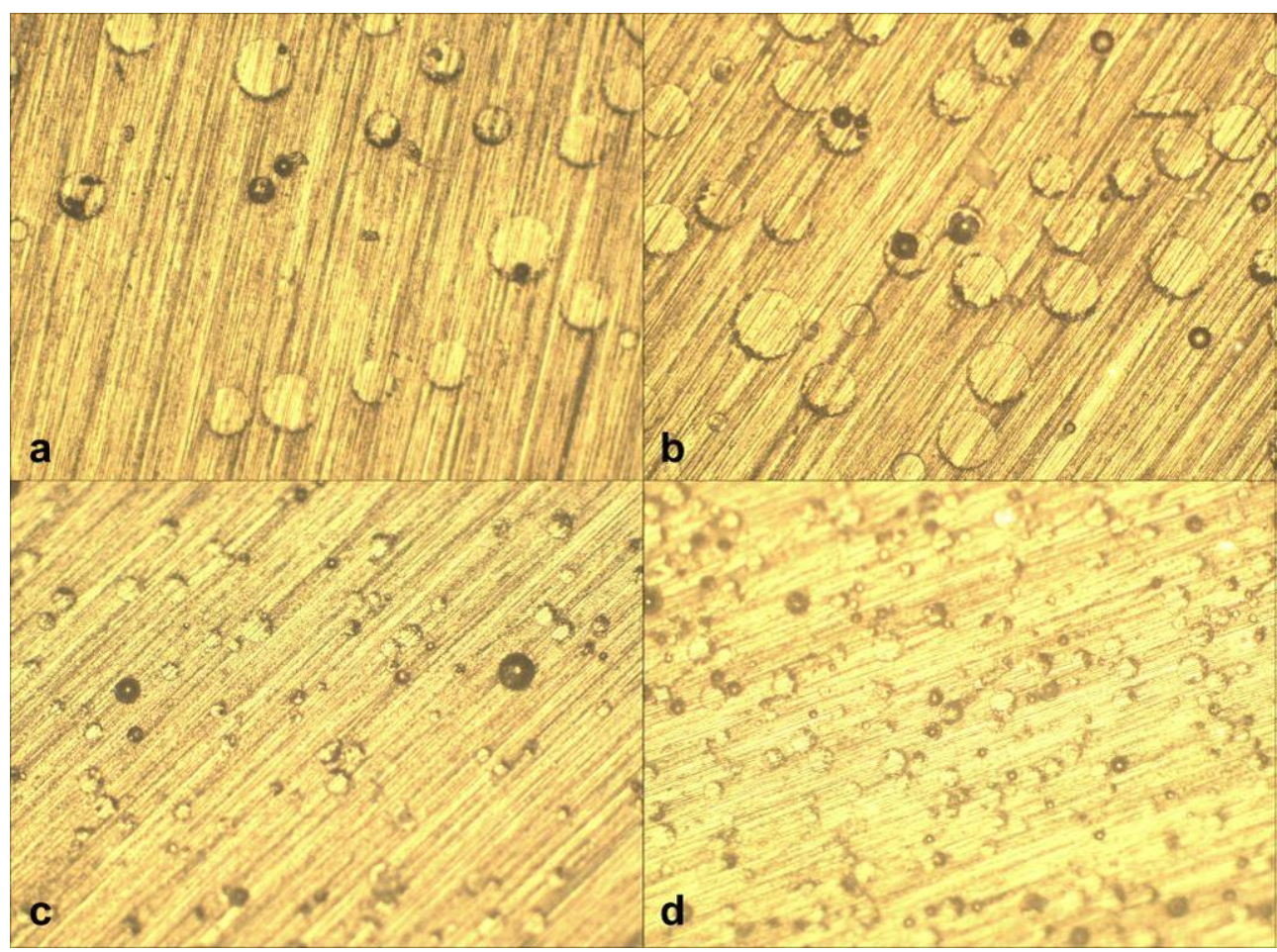

Figure 1. 50x magnification optical micrographs of epoxy-glass composites having different sphere diameter and volume content: a) $100 \mu \mathrm{m}, 10$ vol- $\%$; b) $100 \mu \mathrm{m}, 30$ vol- $\%$; c) $25 \mu \mathrm{m}$, 10 vol- $\%$; d) $25 \mu \mathrm{m}, 30$ vol- $\%$.

SAN, glass-filled SAN and ABS materials were compression moulded into sheets by using a laboratory scale press. First, the materials were preheated for 8 minutes at $190^{\circ} \mathrm{C}$. Then, they were moulded for 7 minutes under a pressure of 40 bar and for another 7 minutes under a pressure of 100 bar. Finally, they were brought to room temperature by letting cool water flow in the cooling system of the press.

Table 1 Matrix and filler type, size and amount in the composite materials

\begin{tabular}{|c|c|c|c|c|}
\hline \multirow{2}{*}{ Matrix } & \multicolumn{4}{|c|}{ Filler } \\
\cline { 2 - 5 } & & type & diameter $(\mu \mathrm{m})$ & content (vol-\%) \\
\hline \multirow{3}{*}{ Epoxy } & none & - & - & - \\
\cline { 2 - 5 } & glass spheres & \multirow{2}{*}{ hard } & 100 & \multirow{2}{*}{$10,20,30,40$} \\
\cline { 2 - 5 } & Fortegra 100 & soft & 25 & $5,10,20,30,40$ \\
\cline { 2 - 5 } & none & - & - & - \\
\cline { 2 - 5 } SAN & glass spheres & hard & 25 & $10,25,40$ \\
\cline { 2 - 5 } & SAN-grafted polybutadiene & soft & 1 & \multicolumn{2}{|c}{1} \\
\hline
\end{tabular}

Filler type, size and volume content of the composites are summarized in Table 1. Volume content was calculated from weight content using (apparent) filler density data as provided by the supplier. In the case of ABS the volume content of the sole polybutadiene rubber contained in the dispersed phase of SAN-grafted polybutadiene was considered as significant and equivalent to the weight content of polybutadiene specified by the producer. 


\subsubsection{Test specimen preparation}

For all materials the surface of the samples subjected to scratch testing was one of the faces contacting the mould during casting/moulding. Prior to testing, the surface was cleaned with commercial detergents and the specimen dried in a vacuum oven at $50^{\circ} \mathrm{C}$ for 12 hours.

Specimens for mechanical testing were machined to the required dimensions by milling.

\subsection{Methods}

All the tests were performed at a temperature of $23^{\circ} \mathrm{C}$, with at least three repetitions for each test/condition.

\subsubsection{Dynamic Mechanical Analysis (DMA)}

The tests were carried out in a three-point bending configuration by using a TA Rheometric Series RSA III instrument. Specimens measured $7 \mathrm{~mm}$ in width, $2 \mathrm{~mm}$ in thickness, with a span of $40 \mathrm{~mm}$. Storage modulus was measured by applying a sinusoidal strain of $6 \cdot 10^{-5}$ amplitude in the frequency range $0.01-50 \mathrm{~Hz}$.

\subsubsection{Compression Test}

Cylindrical epoxy-based composite samples measuring $14 \mathrm{~mm}$ in diameter and $15 \mathrm{~mm}$ in height and cubic SAN-based composite samples with an edge length of $9 \mathrm{~mm}$ were prepared for compression testing. The tests were carried out by an Instron 1185R5800 dynamometer fitted with compression plates at constant crosshead speeds of $0.1,1$ and $10 \mathrm{~mm} / \mathrm{min}$. To reduce friction between the test specimen and the plates and so avoid barrelling of test specimens a $50 \mu \mathrm{m}$ thick polytetrafluoroethylene film was put between the sample faces and the metal plates. The displacement between the two plates was measured using a strain-gage extensometer. The maximum of the stress-strain curves was taken as an apparent, conventional value of the yield stress.

\subsubsection{Scratch Test}

Specimens of $30 \mathrm{~mm}$ in length and $15 \mathrm{~mm}$ in width were obtained from the $5 \mathrm{~mm}$ thick cast (Epoxy) and $9 \mathrm{~mm}$ thick moulded (SAN) plates.

The tests were performed on a CSM Microscratch tester in load-controlled mode keeping the load constant during the test and selecting the load value so as to obtain the same base penetration depth $(\sim 40 \mu \mathrm{m})$ in all materials. Sliding tip velocity and scratch length were selected as $5 \mathrm{~mm} / \mathrm{min}$ and $4 \mathrm{~mm}$, respectively. The conical indenter had a $120^{\circ}$ apex angle and a spherical tip with $200 \mu \mathrm{m}$ radius.

Three parameters were directly measured during the scratch tests: penetration depth, residual depth and acoustic emission. The penetration depth was determined as the difference between the original profile of the sample surface and the vertical position of the indenter during the test. The residual depth was the groove's depth measured after elastic and viscoelastic recovery occurred.

\subsubsection{Optical Microscopy}

Top view optical microscopy images of the scratch tracks were taken by using an Olympus BX60 optical microscope in reflection mode with $5 \mathrm{x}$ magnification to observe the differences in the scratch deformation modes between neat polymers and their composite counterparts incorporating hard or soft fillers. 


\section{Theory}

One of the most commonly used methods for evaluating the scratch performance is the determination of scratch hardness, which represents an average response of the material to the contact load exerted by the indenter. Scratch hardness is calculated as the normal load divided by the projected load bearing area. The complexity of assessing scratch hardness results from the difficulty in determining the true contact area. The classical approach assumes that only the front half of the indenter is in contact with the material and the radius of this semi-circular contact is taken as half of the residual scratch width. These assumptions are appropriate in the case of materials that deform mainly plastically such as metals but they are not accurate for viscoelastic-viscoplastic materials like polymers: in this case the rear side of the indenter is also partly in contact with the material owing to a substantial elastic and viscoelastic recovery of the deformation impressed by the indenter. Several attempts to estimate the true contact area by taking into account indenter geometry, pile-up formation in front and at the sides of the indenter and strain recovery occurring at the rear side of the indenter are reported [2-4,13].

Pelletier et al [4] suggested a method to predict the true contact area via a parameter called "rheological factor", $X$, which is defined as the ratio of the strain imposed by the indenter to the yield strain of the material [33]. $X$ can be related to the modulus/yield stress ratio of the material and the indenter geometry. For a detailed analysis of the model and for the application of that model on various polymers the reader can refer to the studies of Pelletier et al [4] and Kurkcu et al [34], respectively.

In particular, Kurkcu et al [34] determined the scratch hardness of a series of polymers imposing different loading histories and sliding tip velocities. As knowledge of the material's elastic modulus and yield stress is required to apply this model, in the case of viscoelastic materials the values of the relevant bulk mechanical properties should be determined for a strain rate that is consistent with the strain rate applied during the scratch test. As the latter is usually quite high, an extrapolation from values measured at ordinarily low strain rates is generally required, as discussed in Kurkcu's paper. In the present case, a strain rate of about $0.3 \mathrm{~s}^{-1}$ was estimated to occur during scratch testing.

The authors demonstrated that scratch hardness is independent of the applied loading history (at least for the few cases tested: constant penetration depth, constant load and load increasing at a constant rate) and it is directly proportional to the "apparent" compressive yield stress of the material (Fig. 2). Scratch hardness was also found to correlate very well with the indentation hardness of the investigated materials. The yield stress is referred to here as "apparent" because it was determined according to the conventional definition of the yield point, identified with the point of relative maximum in the loading curve (or engineering stress-strain curve). 


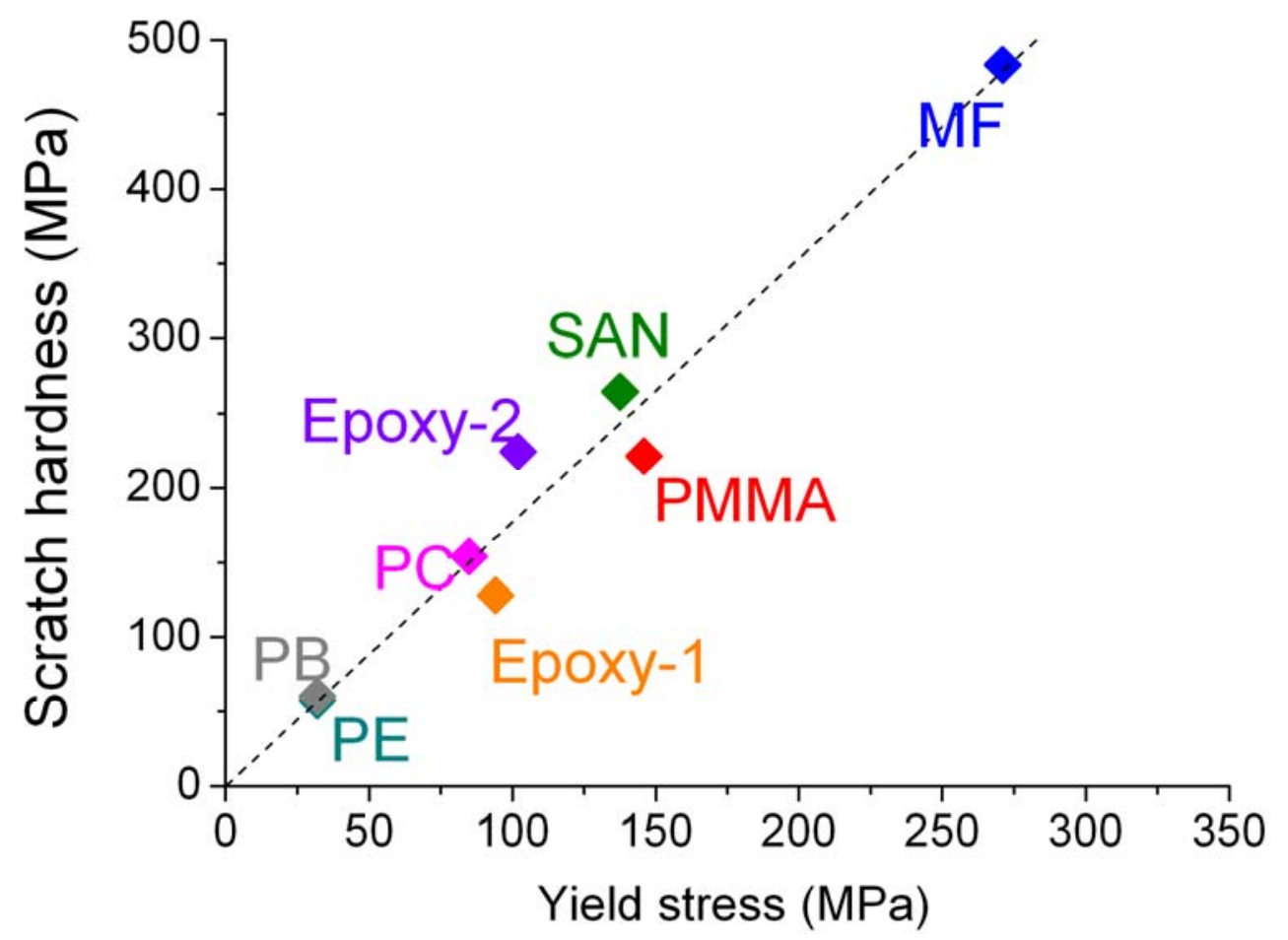

Figure 2. Correlation between scratch hardness and yield stress in neat polymers [34]. The material labelled as "Epoxy-2" is the same epoxy resin investigated in the present study.

In this study, the validity of this correlation in composites containing hard or soft fillers was questioned. The apparent yield stress of particulate-filled composites usually increases or decreases with the incorporation of hard or soft fillers, respectively. It is useful to remark that the yield stress of a composite material is determined from the loading curve of the composite material as if it was homogeneous although local phenomena are significantly more complex, since the stress distribution is altered by the mismatch of the elastic moduli of the particles and the matrix. A rigid particle draws the stress "flow lines" into its inside, thus relieving the surrounding matrix; on the contrary, with a soft particle the stress "flow lines" in the surrounding matrix thicken.

Depending on the relative size of the filler particles and the indenter, the effects of the aforementioned phenomena on the scratch process may or may not be neglected. Whether the apparent yield stress is sufficient or not to describe the scratch behaviour when the local phenomenon is dominant is discussed in the following section.

\section{Results}

The repeatability of DMA tests, compression tests and scratch measurements was generally quite good, with a standard relative error mostly below 5\%. Only in a few cases (mainly with glass-filled composites) a higher value between 5\% and 10\% was reported.

The three quantities measured during the scratch tests, i.e. penetration depth, residual depth and acoustic emission can reveal the differences in scratch behaviour between neat polymers and composites. Acoustic radiations are emitted when the material undergoes some damage triggering a sudden release of elastic energy; hence, these emissions are an indication of material failure. In the case of composites, possible failure mechanisms originating acoustic emissions are matrix cracking, filler breakage and filler-matrix debonding. 


\subsection{Hard Particle Composites}

\subsubsection{Epoxy / Glass Sphere Composites}
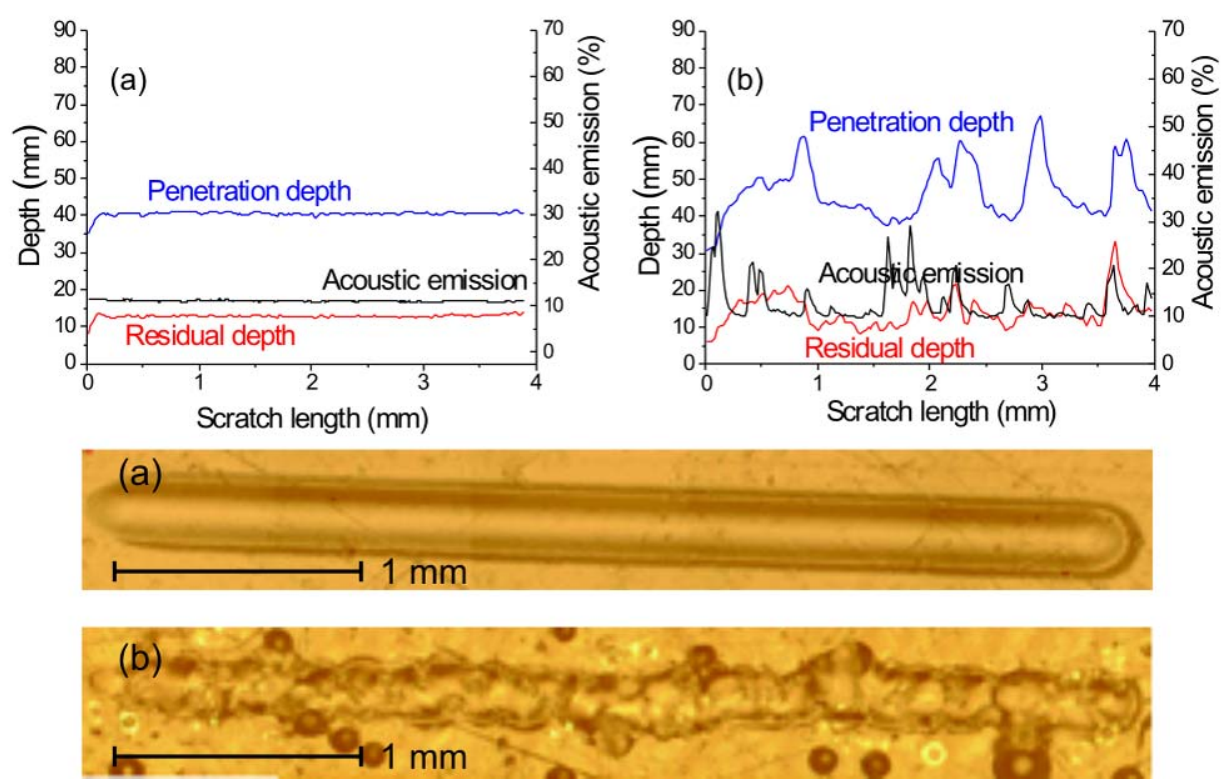

Figure 3. Above: examples of penetration depth, residual depth and acoustic emission records plotted as a function of scratch length. Below: examples of optical micrographs of top views of a scratch groove; (a) neat epoxy and (b) epoxy composite with 40 vol- $\%$. of glass spheres $($ diameter $=100 \mu \mathrm{m})$.

Penetration depth, residual depth and acoustic emission records, together with optical microscope images, demonstrate clearly the difference between scratch behaviour of neat epoxy and that of epoxy-glass sphere composites: see Figures $3 \mathrm{a}$ and $3 \mathrm{~b}$. During scratching, neat epoxy deforms viscoelastically and viscoplastically forming pile-ups both in front and at the sides of the groove left by the indenter. No crack formation at the bottom of the groove is observed. This behaviour is usually referred to as ductile ploughing [2]. The picture alters considerably with the incorporation of glass spheres: while a neat, linear border of the groove is observed in the plain resin case (Figure 3a) an irregular, bamboo-like trace with formation of chips and debris is observed in the case of the composite (Figure 3b). Presumably, when the indenter comes across the glass spheres embedded in the epoxy matrix, it may break them or displace them pushing them aside, or even pass over them without damaging them. The peaks in the acoustic emission curve reveal likely a breakage of the spheres while the peaks in penetration and residual depth curves can be associated to the cavities left by the displacement of the spheres.

Figure 4 (a to d) presents penetration depth, residual depth and acoustic emission records of epoxy composites containing glass spheres of $100 \mu \mathrm{m}$ in diameter in different amounts. It should be emphasized that increasing the filler content four times in volume does not mean that the probability for the indenter of finding a particle on its way also increases that much: as a matter of fact the scratch test is carried out along one direction and the 1D encounter probability is not proportional to the 3D density of particles. Moreover, the quantitative effect of filler content may not appear evidently from these data: since the number of particles the indenter comes across is random when the spheres are well dispersed. Thus, many test repetitions are required to collect statistically significant sets of data. 

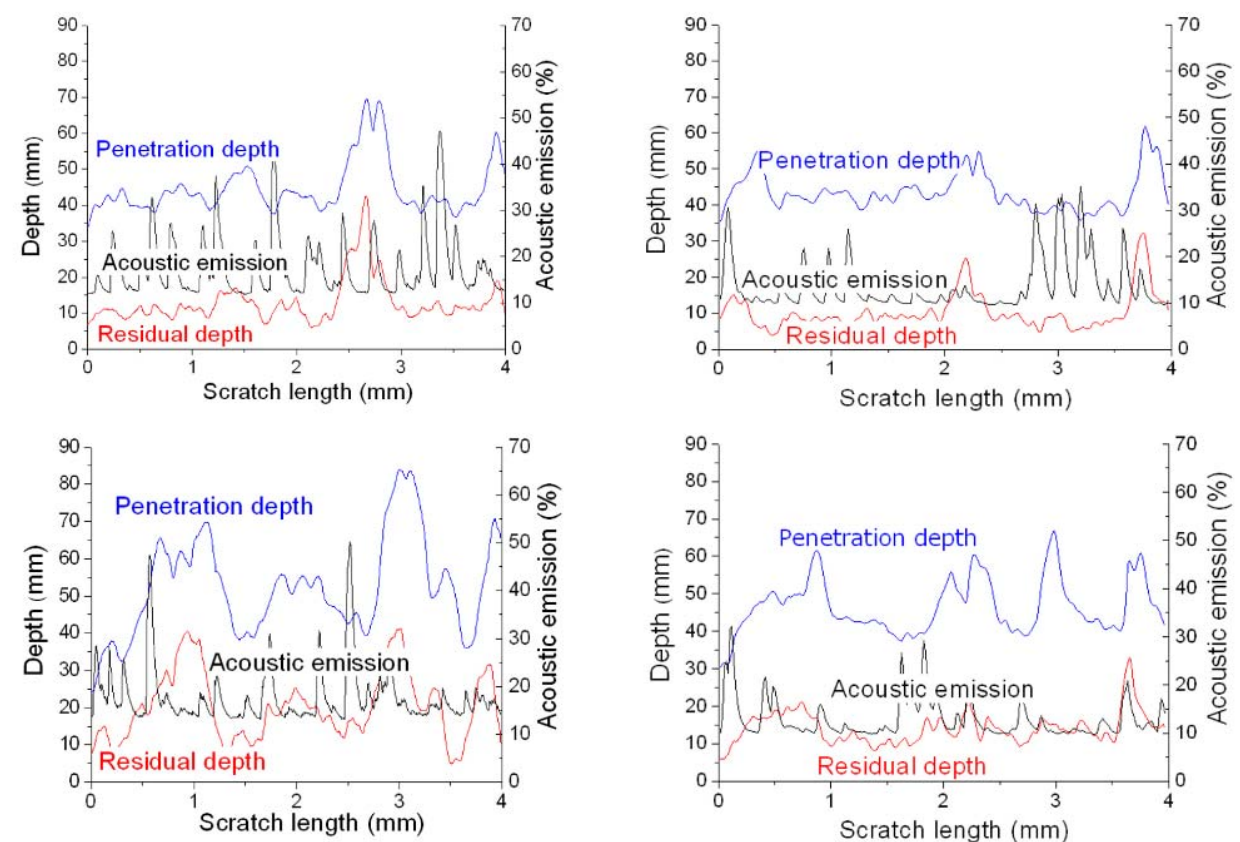

Figure 4. Penetration depth, residual depth and acoustic emission records plotted as a function of scratch length of epoxy-based composites filled with $100 \mu \mathrm{m}$ glass spheres in varying amounts: (a) 10 vol-\%. (b) 20 vol-\%. (c) 30 vol-\%. (d) 40 vol- $\%$.

[Note - Fig. 4(d) identical to Fig. 3(b) above.]
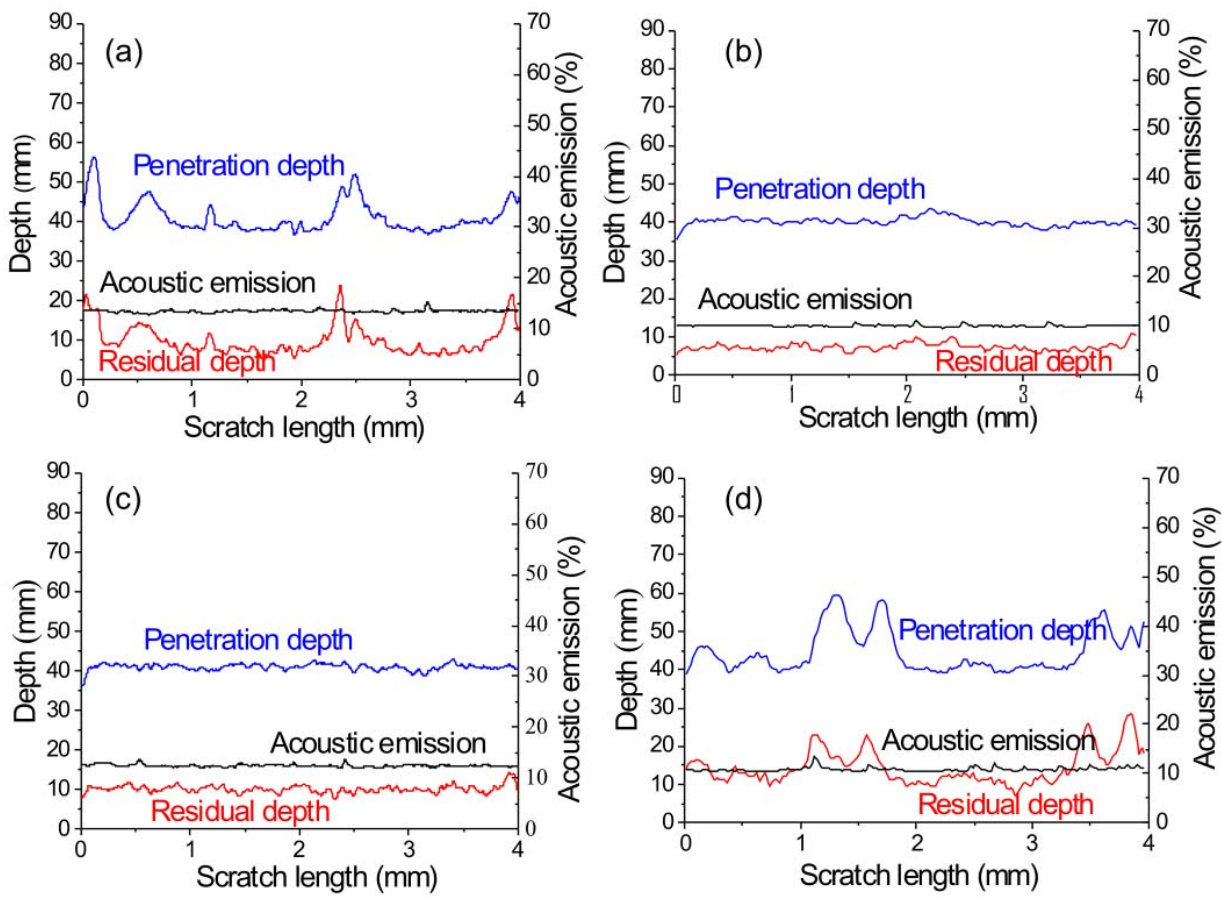

Figure 5. Penetration depth, residual depth and acoustic emission records plotted as a function of scratch length of epoxy-based composites filled with glass spheres having a diameter of $25 \mu \mathrm{m}$ in varying amounts: (a) 10 vol-\%. (b) 20 vol-\%. (c) 30 vol- $\%$. (d) 40 vol- $\%$. 
Figure 5 (a to d) shows penetration depth, residual depth and acoustic emission records obtained on epoxy composites, containing glass spheres of $25 \mu \mathrm{m}$ in diameter in different amounts. By contrast with the case of glass spheres of larger size seen in Figure 4 large peaks are observed neither in the penetration depth nor in the acoustic emission traces. This observation confirms that the previously observed peaks are to be related to the interaction of the indenter with particles of comparable size. Conversely, when the particle size is significantly smaller the composite behaves more similarly to a homogeneous material.

The tensile storage modulus of the epoxy-based composites filled with glass spheres of the two diameters is displayed in Figure 6. The experimental data appear to be independent of filler size, as expected (see e.g. [35]), and only slightly dependent on filler content. The linear fit (dotted line) is just an empirical attempt at describing this dependence, at variance with the theoretical predictions (solid lines).

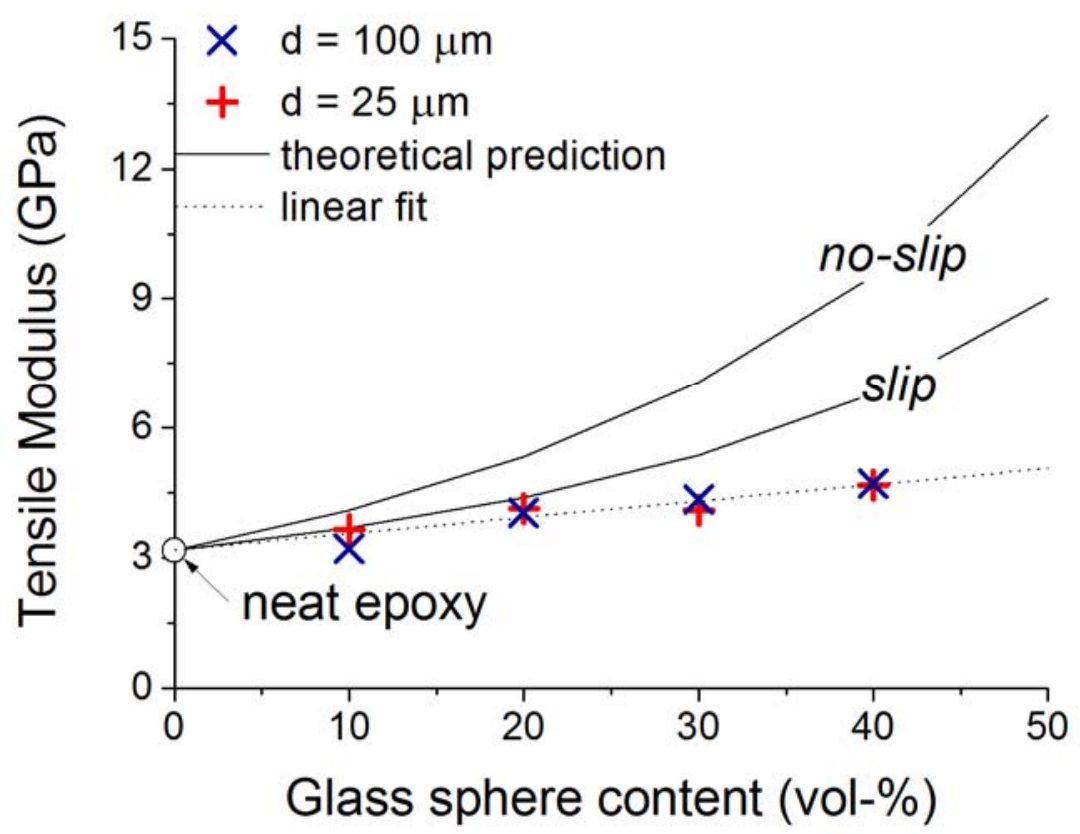

Figure 6. Effect of incorporation of glass spheres on the storage tensile modulus of epoxy-based composites (frequency $=1 \mathrm{~Hz}, d=$ filler diameter). Dashed line: empirical linear fit, continuous lines: theoretical predictions according to the "modified" Nielsen model (see Appendix).

The prediction of the dependence of the (static) elastic moduli on filler content, especially for fillers stiffer than the matrix, is dealt with by a vast literature (e.g. see [35-39] for relevant reviews). Mostly the theoretical treatments, based on different models, assume perfect adhesion at the filler-matrix interface, which often results in overestimating the modulus of the composite. A "modified" Nielsen model allowing for a varying degree of adhesion of the matrix polymer to the filler particles will be considered here [see Appendix for details]. In Figure 6 the predictions of this model for the two extreme cases ('slip' and 'no-slip') are reported as solid lines. The experimental data appear to be even lower than the lowest theoretical prediction ('slip' case).

According to Nielsen [36] experimental data are often erroneously too low (lower than theoretically predicted) because of two other possible reasons: a "skin effect" (i.e. excess of polymer at the surface of most composite test specimens because of the restrictions imposed by the walls of moulds) and residual tensile thermal stresses in the polymer matrix 
surrounding the filler particle (resulting from the mismatch of the thermal expansion coefficients of the polymer and filler). The former is not the case in the present work since specimens for mechanical testing were machined from larger moulds, thus removing their polymer-rich surface layers. The latter possible cause of error cannot completely be excluded, however, in view of the thermal treatment (post-curing at $100^{\circ} \mathrm{C}$ ) undergone by the samples.

In Figure 7, the apparent overall yield stress measured in compression (as defined under section 2.2.2) on the same samples is shown. Evidently the incorporation of glass spheres barely affects this property, independently of the filler size. The linear fit (dashed line) is just an empirical attempt at describing the slight variation with filler content. No comparison with theoretical predictions was attempted. One can just observe that, as yield stress is expected to increase with hard filler content due to the stress concentration effects produced by the difference in modulus, the slightly opposite result can be explained with the same arguments that may explain the values in modulus resulting lower than theoretically predicted.

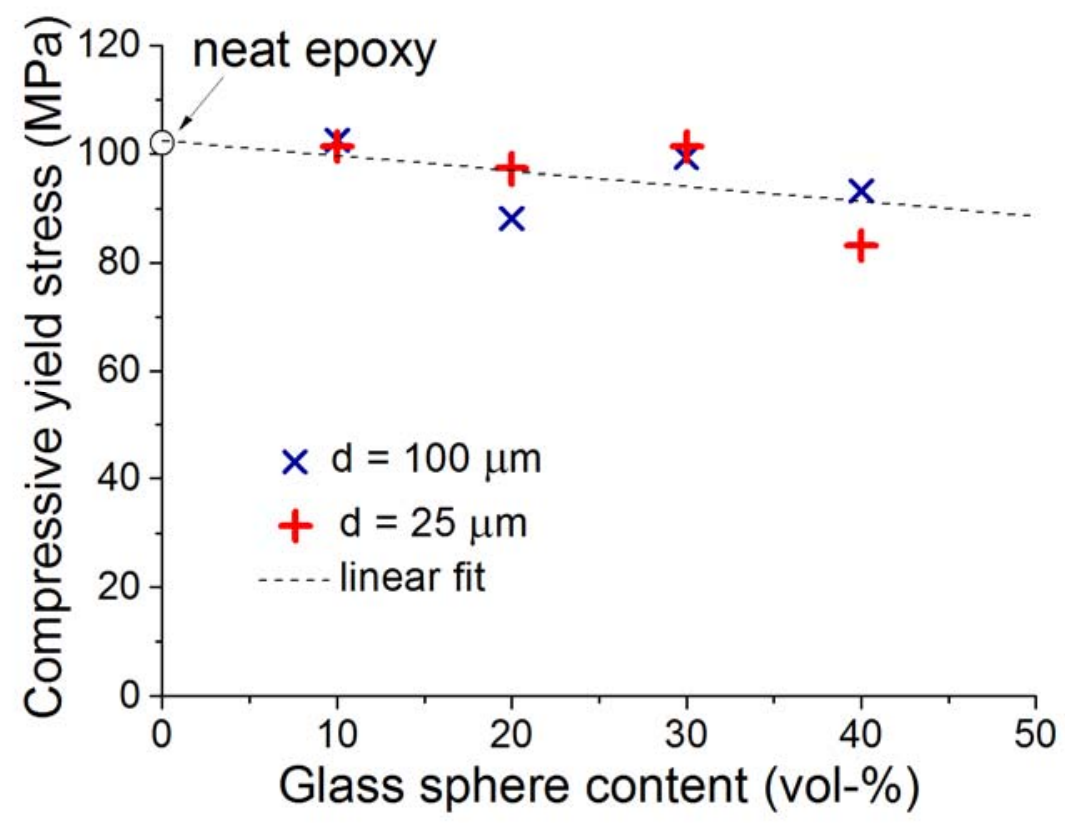

Figure 7. Effect of incorporation of glass spheres on the yield stress in compression of epoxy based composites (strain rate: $10^{-4} \mathrm{~s}^{-1}, d=$ glass sphere diameter).

Scratch hardness of the composites was determined from modulus and yield stress data as suggested by the Pelletier's scratch model and the validity of the relation between the scratch hardness and the yield stress, which was observed for neat polymers, was questioned. Figure 8 displays the data for glass sphere-epoxy composites together with the previous correlation observed in [34] for neat polymers. It can be immediately seen that the presence of glass spheres increases the composites scratch hardness, despite filler not having a considerable effect on their yield stress. The increase in scratch hardness could be attributed to the larger modulus of the composites; yet the increase in modulus is identical for the two particle sizes examined (Figure 6) while the increase in hardness is greater with the $100 \mu \mathrm{m}$ glass spheres. Moreover, while the modulus increases regularly with increasing filler content (Figure 6) no trend was observed on the scratch hardness with varying filler content. Therefore, it must be concluded that the effect of the filler in this case is not directly related to a change in the overall bulk mechanical properties of the composite. 
It should be emphasized that the reported increase in scratch hardness does not necessarily reflect an overall improvement. In fact, incorporation of rigid particles causes a change in the scratch deformation mode from ductile ploughing to brittle fracture, which may result in a worse appearance effect: scratch visibility increases.

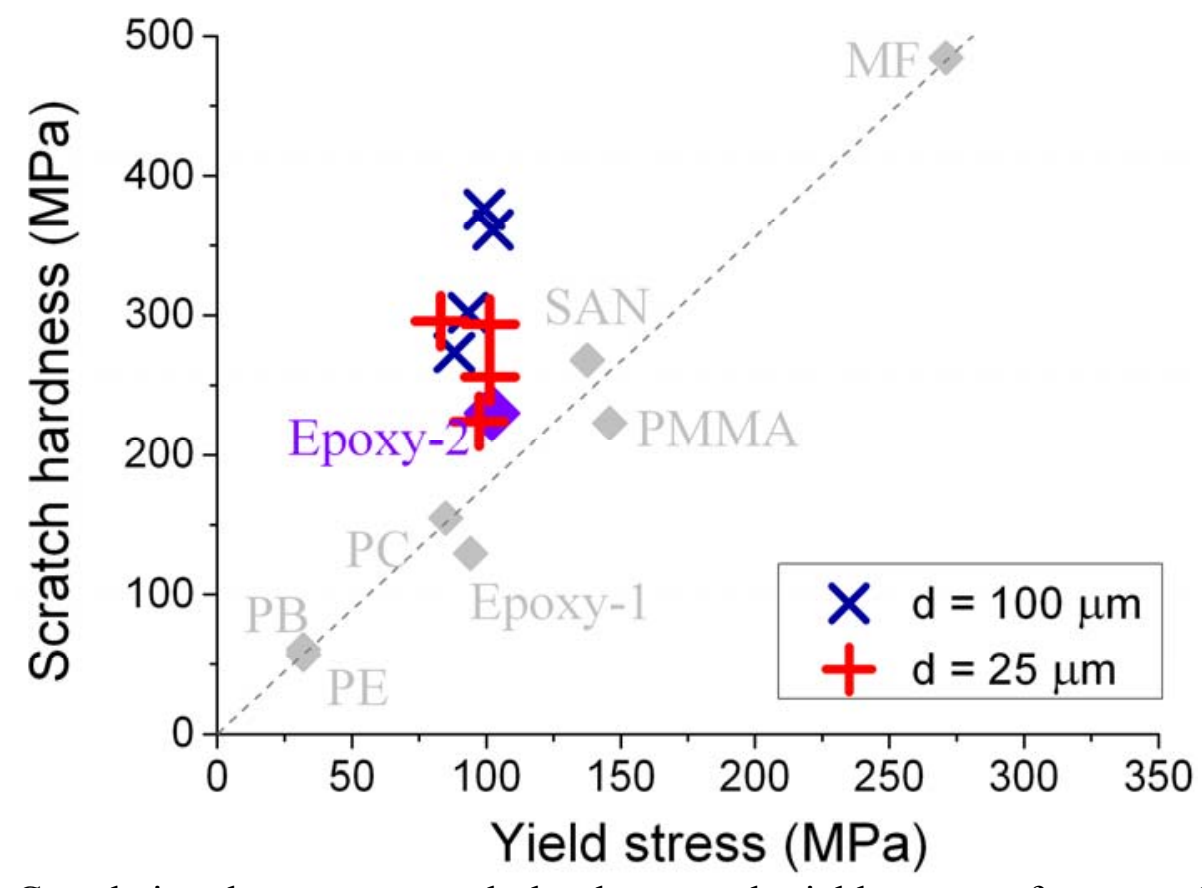

Figure 8. Correlation between scratch hardness and yield stress of epoxy-glass sphere composites. Greyed points correspond to data for neat polymers reported in [34].

\subsubsection{SAN / Glass Sphere Composites}

Incorporation of glass spheres brings about a variation in the scratch deformation mode of neat SAN similar to the one observed for epoxy, as shown in Figure 9. While with neat SAN ductile ploughing occurs, the incorporation of rigid fillers changes the deformation mode to brittle with substantial debris formation. In presence of rigid particles, large peaks are observed in the acoustic emission curves owing to particle breakage. Small peaks can be observed in the depth curves as well, probably due to the voids formed when the particles are displaced. 

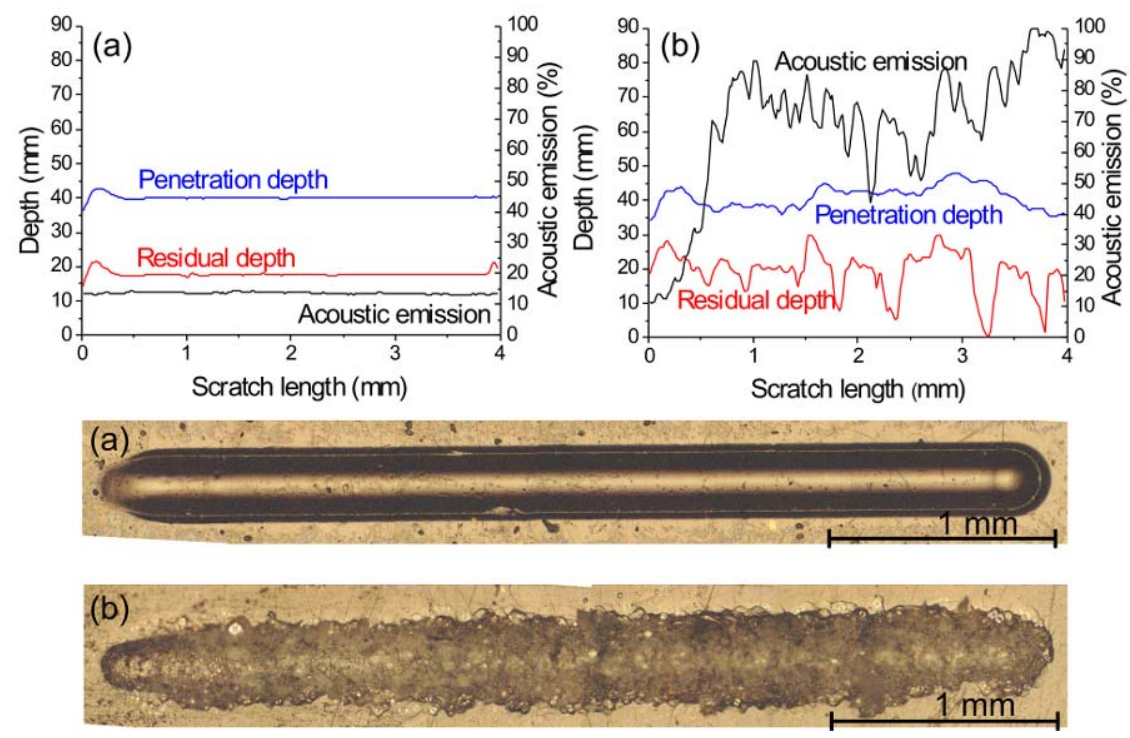

Figure 9. Above: examples of penetration depth, residual depth and acoustic emission records and, below: examples of optical micrographs of top view of a scratch groove, for (a) neat SAN and (b) SAN composite containing 40 vol- $\%$ of glass spheres (diameter $=25 \mu \mathrm{m}$ ).

The change in scratch behaviour of SAN-based composites with varying filler content is displayed in Figure 10. Both the depth curves and the acoustic emission curves show that the effect of the filler is relatively limited at a low content $(10$ vol- $\%)$ but it increases considerably for higher values. 

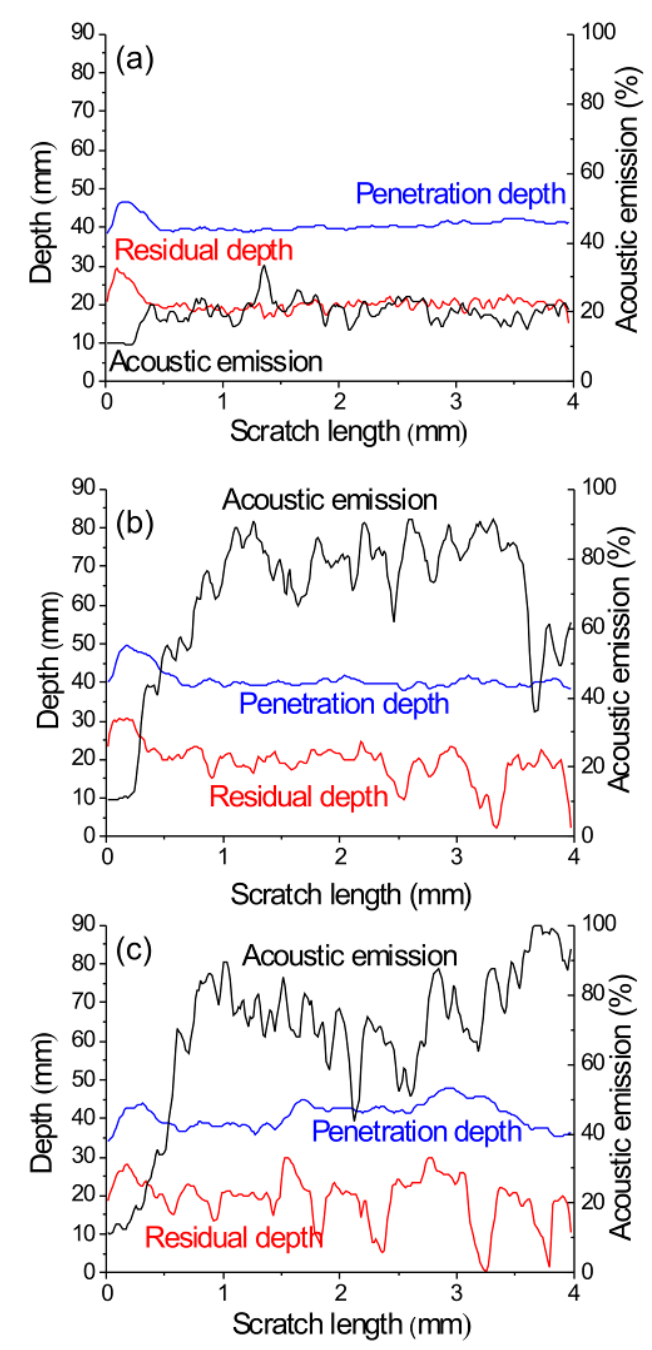

Figure 10. Penetration depth, residual depth and acoustic emission records of SAN-based composites filled with glass spheres having a diameter of $25 \mu \mathrm{m}$ in amounts of: (a) $10 \mathrm{vol}-\%$, (b) 25 vol- $\%$ and (c) 40 vol- $\%$.

Variations of storage modulus and yield stress of SAN with addition of glass spheres are shown in Figure 11 and Figure 12, respectively. The hard filler appears to have no effect on the yield stress of SAN whereas a considerable enhancement is observed in modulus, which increases quadratically with increasing filler content. Unlike epoxy, SAN gives modulus results that are very close to the prediction of the modified Nielsen model (see Appendix) for the case of 'no-slip', which is indicative of a better interaction between the polymer matrix and the glass spheres although in both cases the glass spheres were used as received, without applying any particular seizing or treatment during composite preparation to promote their adhesion to the polymer matrix. As to the yield stress, the same arguments adduced above for the epoxy composites case can be brought forward here. Indeed the difference between the theoretical trend (increasing) and the experimental result (non-decreasing) is less here, which is consistent with the smaller difference between experimental values and theoretical predictions observed for the modulus. 


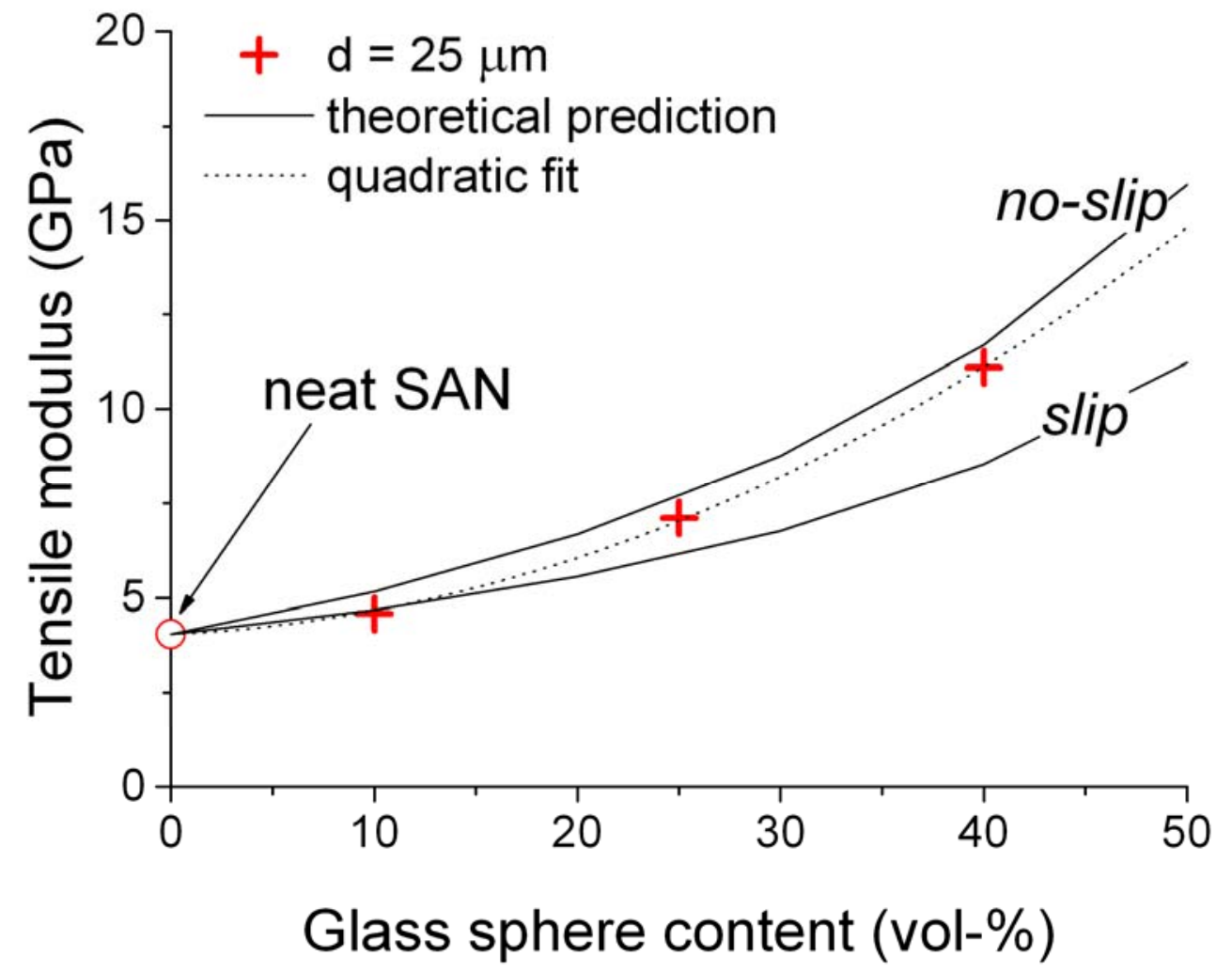

Figure 11. Effect of incorporation of glass spheres on the storage modulus of SAN at $1 \mathrm{~Hz}$. Dotted line: empirical fit (quadratic). Solid lines: theoretical predictions according to the "modified" Nielsen model (see Appendix).

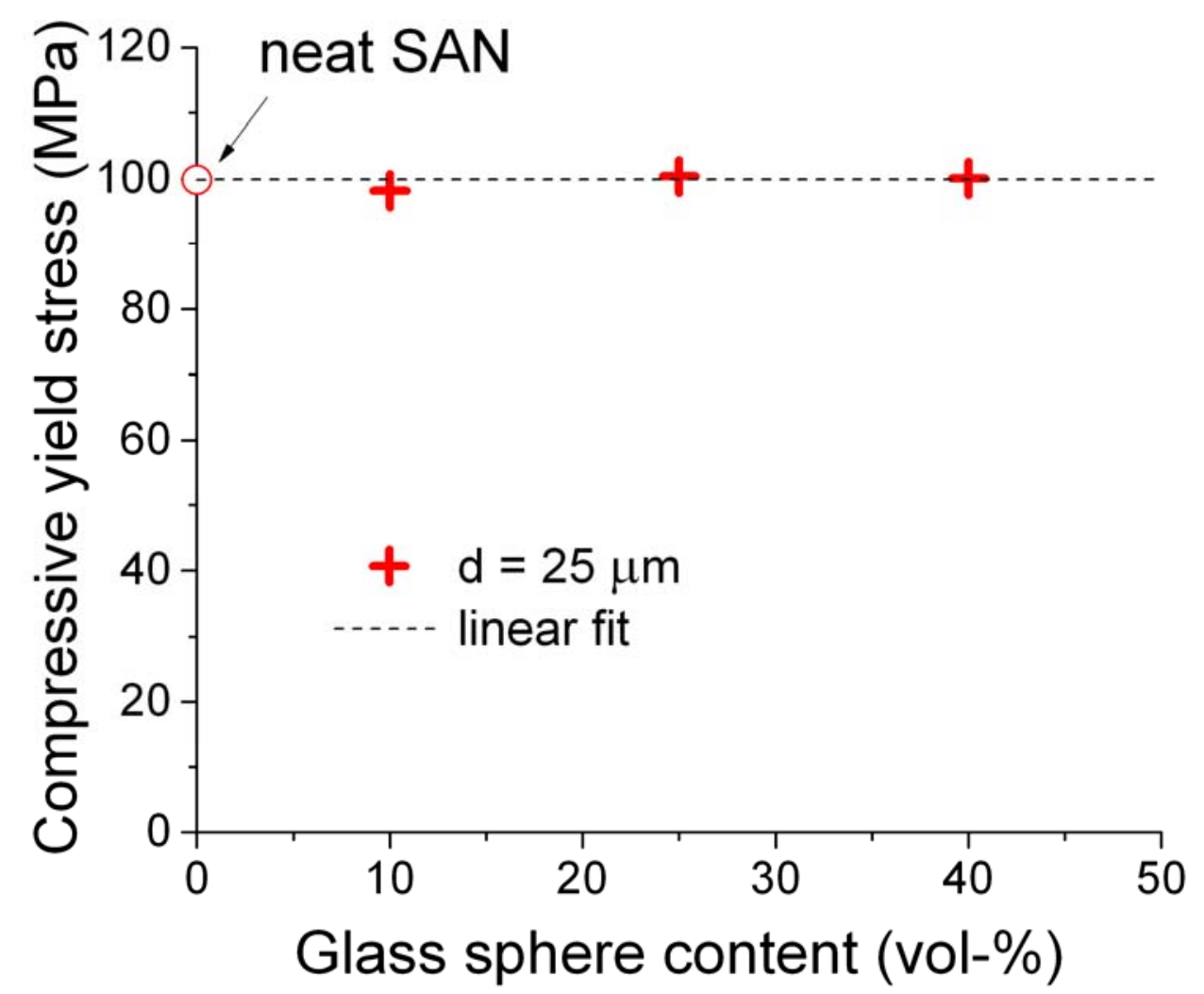

Figure 12. Effect of incorporation of glass spheres on the yield stress of SAN (strain rate: $\left.10^{-4} \mathrm{~s}^{-1}\right)$. Dashed line: empirical fit (linear). 
No attempt was done to compare experimental yield stress values with theoretical predictions. The dashed line in Figure 12 is just an empirical fit (linear).

Figure 13 illustrates the correlation between the scratch hardness and the yield stress of the composites. As in the case of epoxy / glass sphere composites, incorporation of hard fillers results in a scratch hardness enhancement. In this case, however, scratch hardness displays a regular increasing trend with increasing filler content. The presence of hard fillers does not cause a significant variation in yield stress; the reported increase in scratch hardness seems to be caused either by a direct effect of the filler, unrelated to the composites' bulk properties, or by the modulus increase.

Also in SAN / glass sphere composites the scratch visibility is increased by the change in the deformation mode from ductile ploughing to brittle failure but this worsening effect is at least partially balanced by an improvement in the material's resistance to scratch penetration.

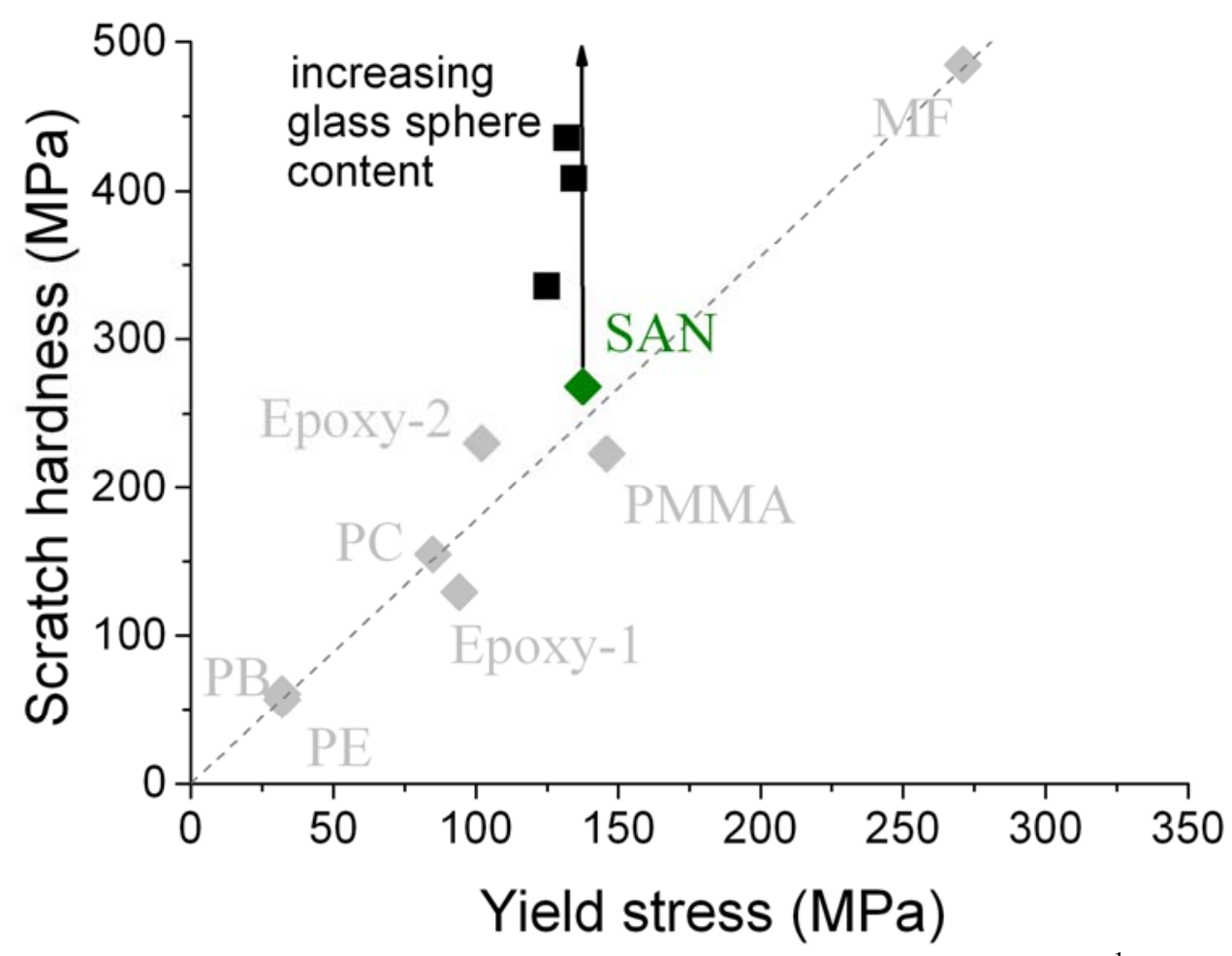

Figure 13. Correlation between scratch hardness and yield stress (at $0.3 \mathrm{~s}^{-1}$ strain rate) of SAN / glass sphere composites. Greyed points correspond to data for neat polymers reported in [34].

\subsection{Soft Particle Composites}

\subsubsection{Epoxy / Soft particle composites}

Figure 14 (a to f) illustrates penetration, residual depth and acoustic emission curves of neat and toughened epoxies. Differently from the case of rigid particles, incorporation of soft particles does not change the scratch deformation mode of the neat polymer: both in neat and toughened epoxy samples ductile ploughing is observed, without any crack formation. This result might be justified by the fact that in this case the particle size (about $1 \mu \mathrm{m}$ ) was much smaller than the indenter radius. 
Yet, although the mode is not changed the scratch behaviour turns out to be strongly affected by the addition of the toughening agent beyond a certain amount. As long as its volume content does not exceed $10 \%$, the composite qualitatively behaves like neat epoxy and no peaks are observed in the depth curves. When the filler content reaches $20 \%$, strikingly large oscillations are observed both in penetration and residual depth curves; they also leave a visible trace in the scratch groove width (see Figure 14, below). However, as the filler content is further increased, these large oscillations disappear, leaving room to much smaller peaks. This very peculiar behaviour may be attributed to a phase inversion occurring at concentrations between 10 and $20 \%$, as indicated by both modulus and yield stress results.
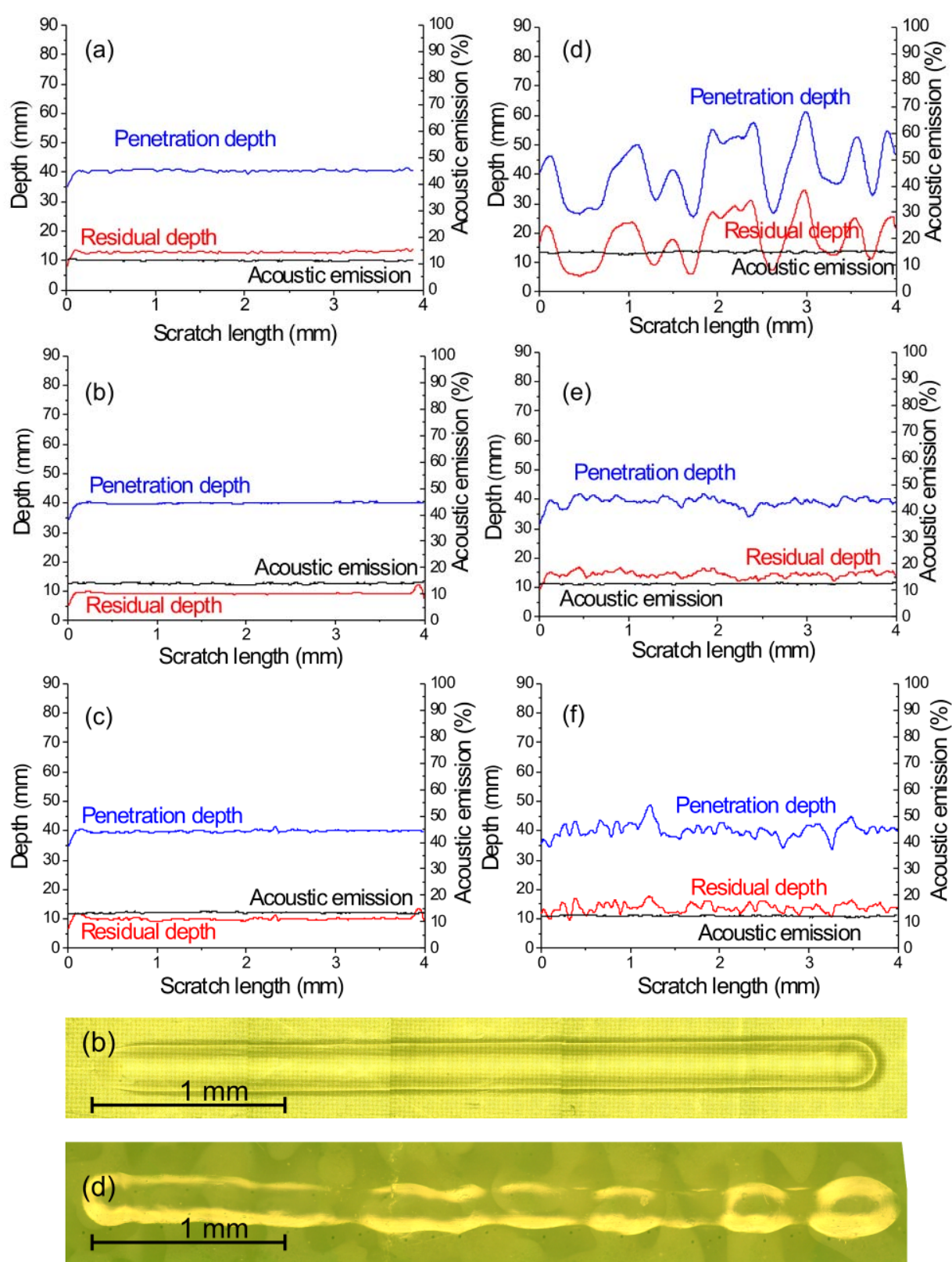

Figure 14. Above: examples of penetration depth, residual depth and acoustic emission records of neat (a) and toughened epoxy containing 5 vol- $\%$ (b), 10 vol- $\%$ (c), 20 vol- $\%$ (d), 30 vol-\% (e) and 40 vol-\% (f) of toughening agent. Below: examples of optical micrographs of top view of scratch grooves for toughened epoxies containing 5 vol- $\%$ (b) and 20 vol- $\%$ (d) of toughening agent. 


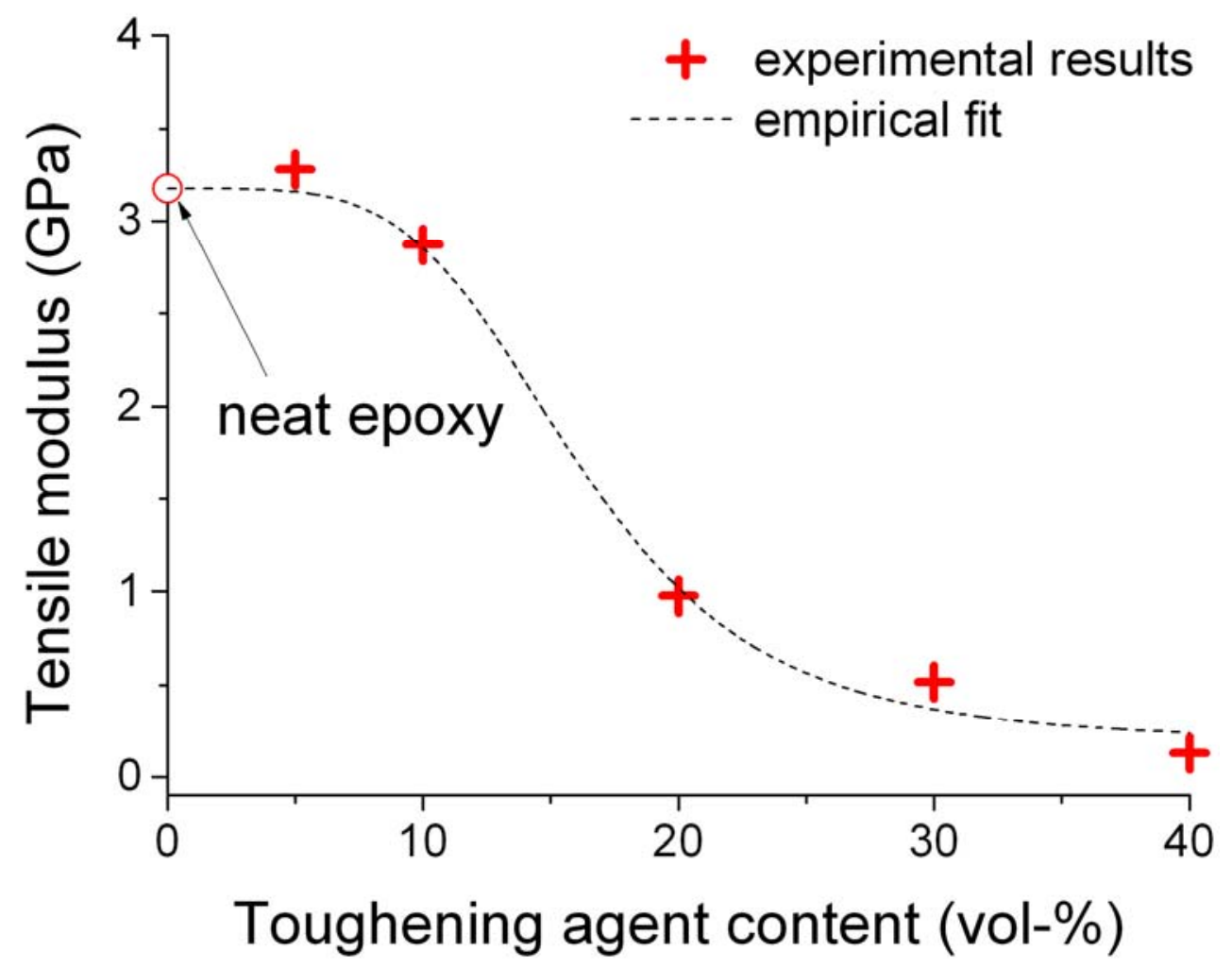

Figure 15. Effect of toughening agent content on the modulus of epoxy composites. Dashed line: empirical fitting (Equation 1 with $x_{0}=16.1 \pm 0.9 ; p=4.4 \pm 0.9$ ).

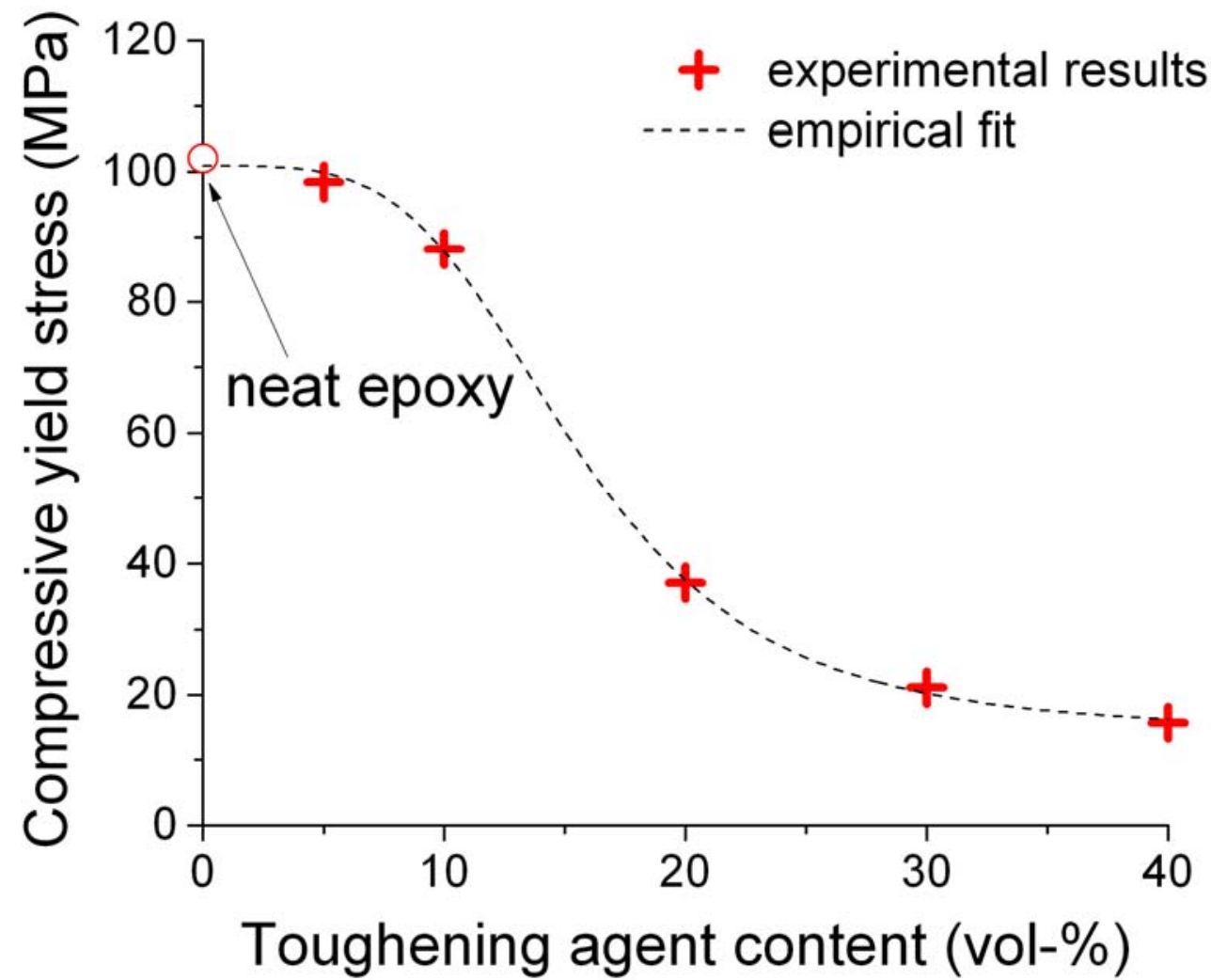

Figure 16. Effect of toughening agent content on the yield stress of epoxy composites. Dashed line: empirical fit (Equation 1 with $x_{0}=15.4 \pm 0.4 ; p=3.9_{ \pm} 0.3$ ). 
Figures 15 and 16 show the effect of the addition of the soft filler on the modulus and the yield stress of epoxy, respectively. Both properties decrease as the toughening agent content increases, as expected. However while the theories predict a gradual reduction in both mechanical properties with increasing content of the soft filler, generally with a curvature of constant sign over the entire composition range, here the experimental results show an inverse S-shaped trend. Both modulus and yield stress change slightly for relatively low contents of the soft component, up to $10 \%$; above this value they decrease drastically up to reaching $20 \%$ and then exhibit a further, moderate decrease which appears to almost level off at the highest filler contents. This behaviour is characteristic of two-phase systems undergoing a phase inversion in their morphology and was not unexpected here, since the toughening agent used is quoted as a 'liquid' by the producer, with no indication of irreversible cross-linking upon incorporation into the epoxy. Both modulus and yield stress vs. volume content data were fitted to the following empirical equation:

$$
y=A_{2}-\frac{A_{2}-A_{1}}{1+\left(x / x_{0}\right)^{p}}
$$

with very similar values of the parameters $x_{0}$ and $p$ for the two quantities.

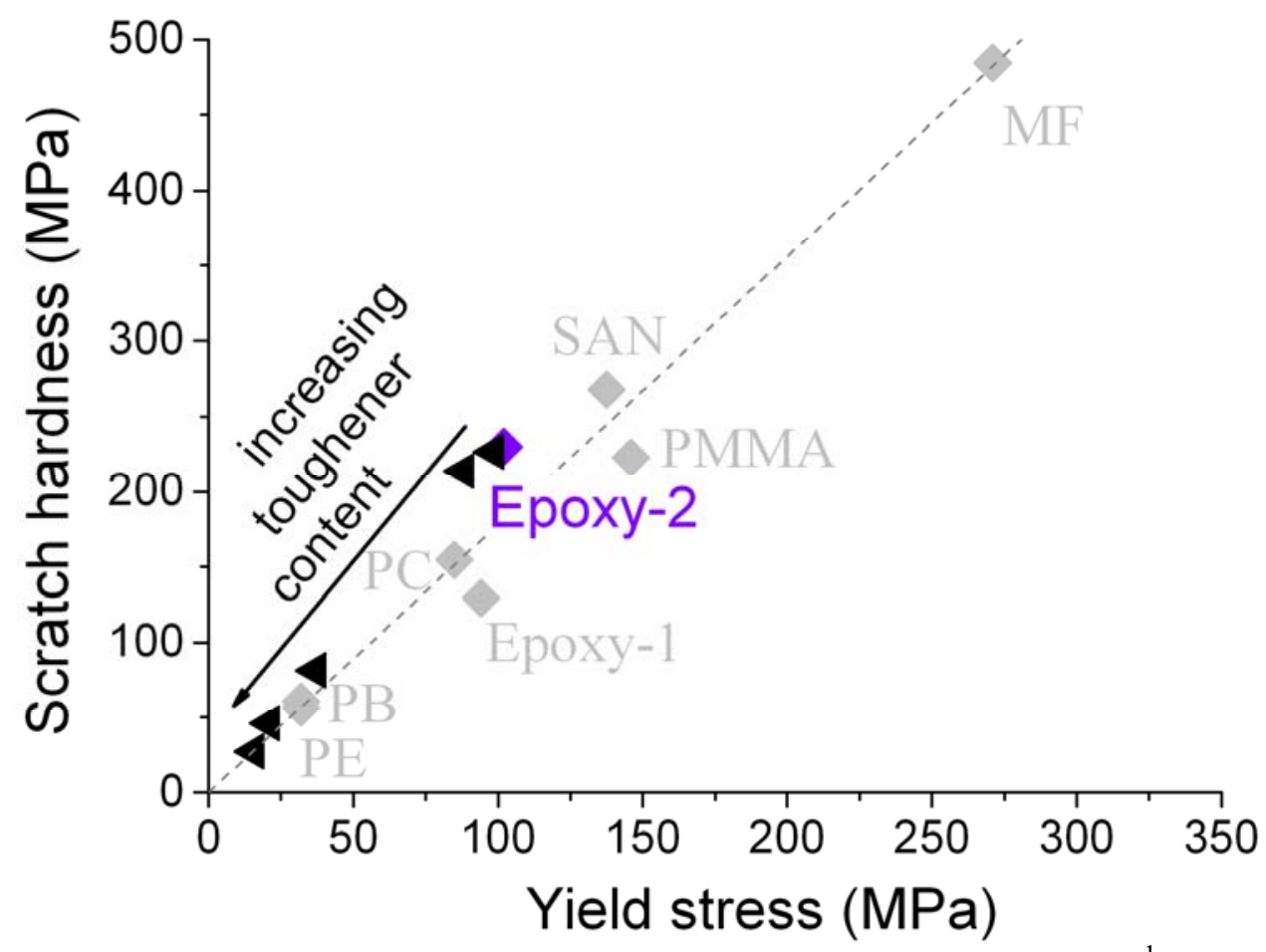

Figure 17. Correlation between scratch hardness and yield stress (at $0.3 \mathrm{~s}^{-1}$ strain rate) of toughened epoxy. Greyed data points correspond to data reported in [34].

Figure 17 illustrates the correlation between the scratch hardness and the yield stress of toughened epoxy. Regardless of the previously described transition occurring at about $16 \%$ volume content, toughened epoxies show a uniform trend, closely following the behaviour already observed for neat polymers. In other words, the compressive yield stress of the material appears again to be the controlling factor in determining scratch hardness, which steadily decreases with increasing soft filler content. However, the dramatic effect of the aforementioned transition dominates the whole mechanical behaviour, with an almost identical effect on all the considered mechanical properties (modulus, yield stress, scratch hardness), as demonstrated in Figure 18. This peculiar behaviour ought to be investigated further. 


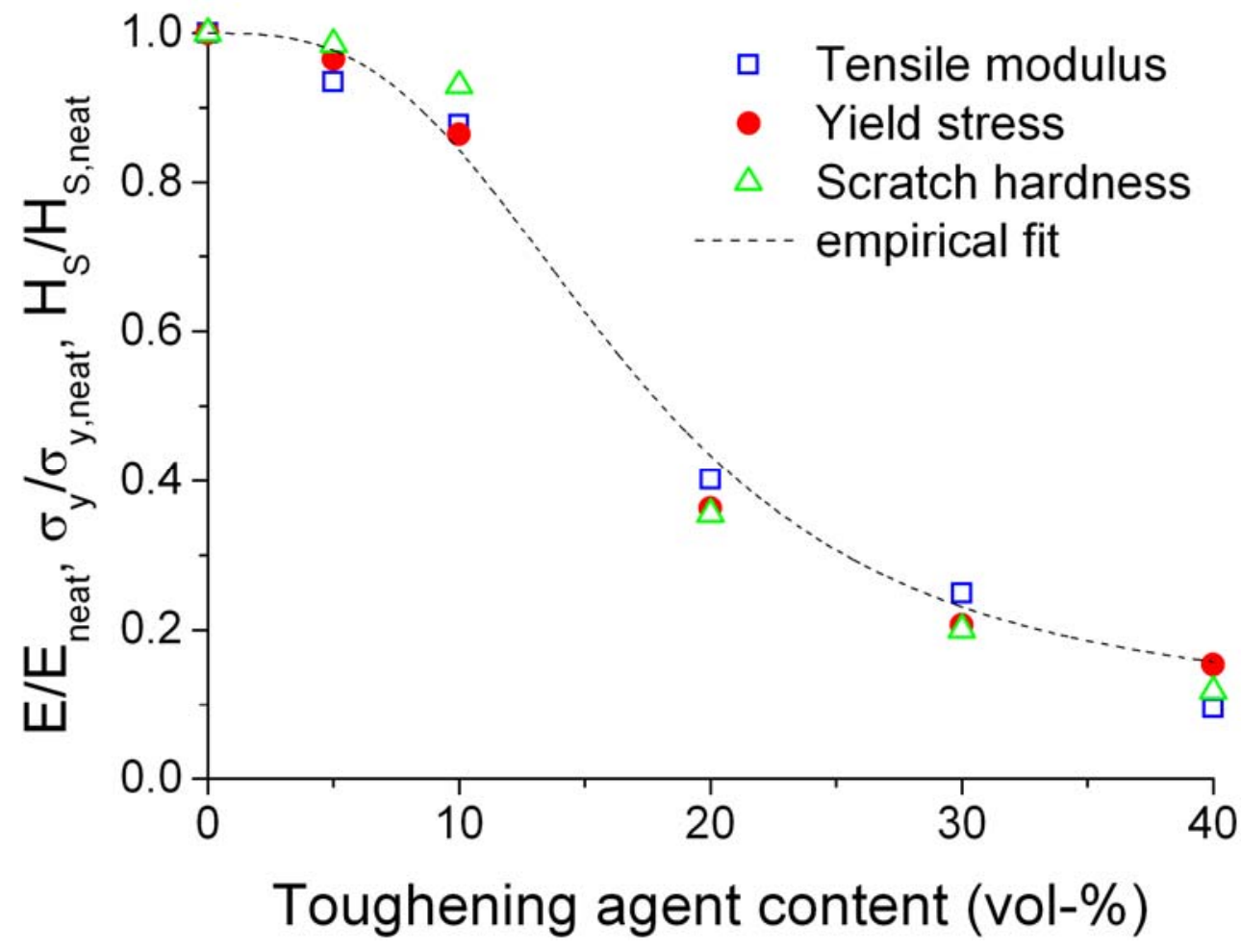

Figure 18. Effect of toughening agent content on the normalized (with respect to the neat polymer value) elastic modulus $E$, yield stress $\sigma_{\mathrm{y}}$ and scratch hardness $H_{\mathrm{S}}$ of toughened epoxy. Dashed line: empirical fit (Equation 1 with $x_{0}=16.8 \pm 1.0 ; p=3.0 \pm 0.5$ ). 


\subsubsection{SAN / Rubber Composites}

Similar to what happens for toughened epoxy, incorporation of soft filler does not modify the scratch deformation mode of SAN. Both in the neat SAN and SAN/rubber composites, ductile ploughing without any crack formation takes place. No peaks are seen in the recorded traces of depth and acoustic emission of neat SAN and SAN/rubber composites, as seen in Figure 19.
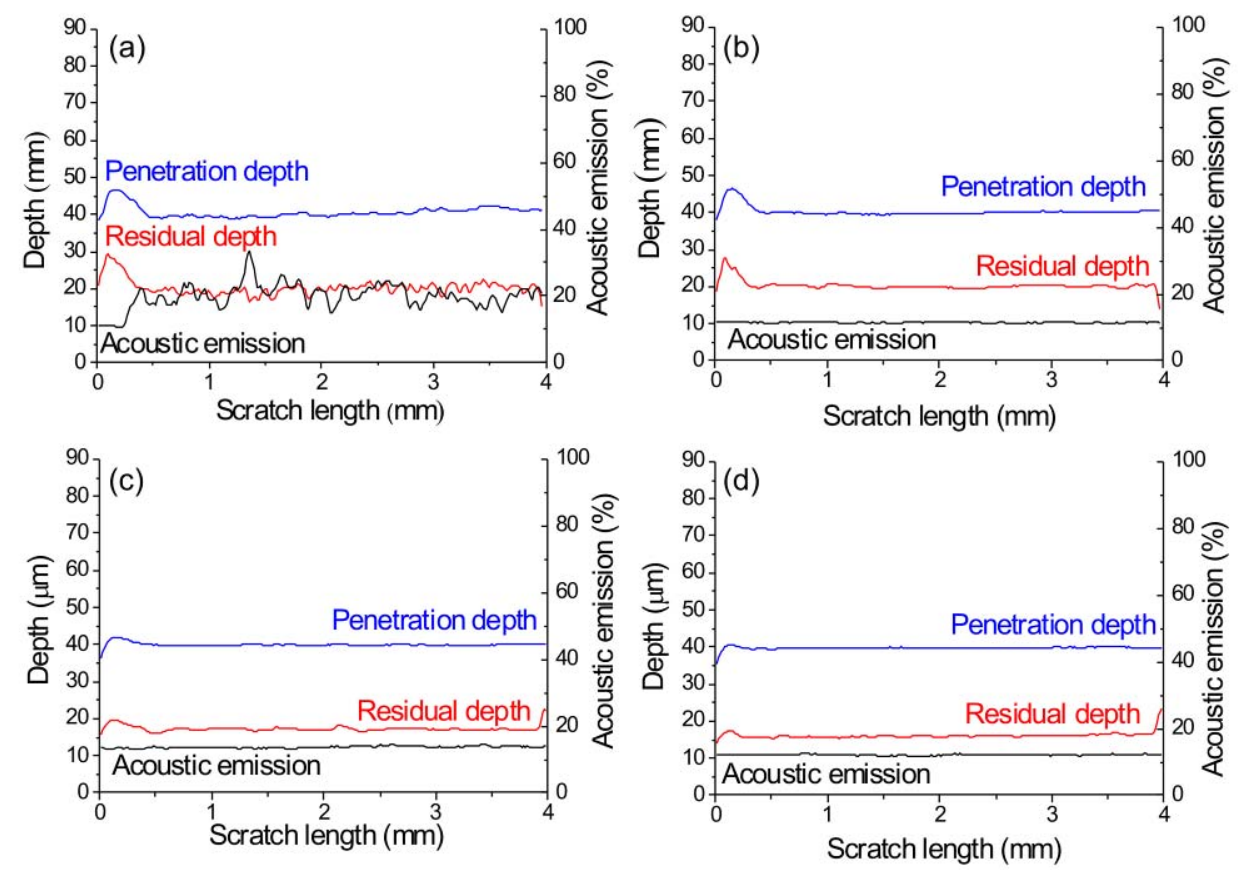

Figure 19. Penetration depth, residual depth and acoustic emission records of neat (a) and rubber-toughened SAN including 10 vol-\%. (b), 25 vol-\%. (c) and 50 vol-\%. (d) of rubber.

Both modulus and yield stress decrease with the incorporation of rubber particles as shown in Figures 20 and 21, respectively. No attempt was done to compare the experimental data of the modulus to some of the theoretical equations proposed in the literature (the dashed line in Fig. 20 is a purely empirical fit) while the experimental data for the yield stress are compared with the predictions of Ishai and Cohen [40] theory (see Appendix) in Fig. 21: the experimental results are somewhat lower than the predicted values. An effective rubber phase volume larger than the nominal rubber content could explain the difference. 


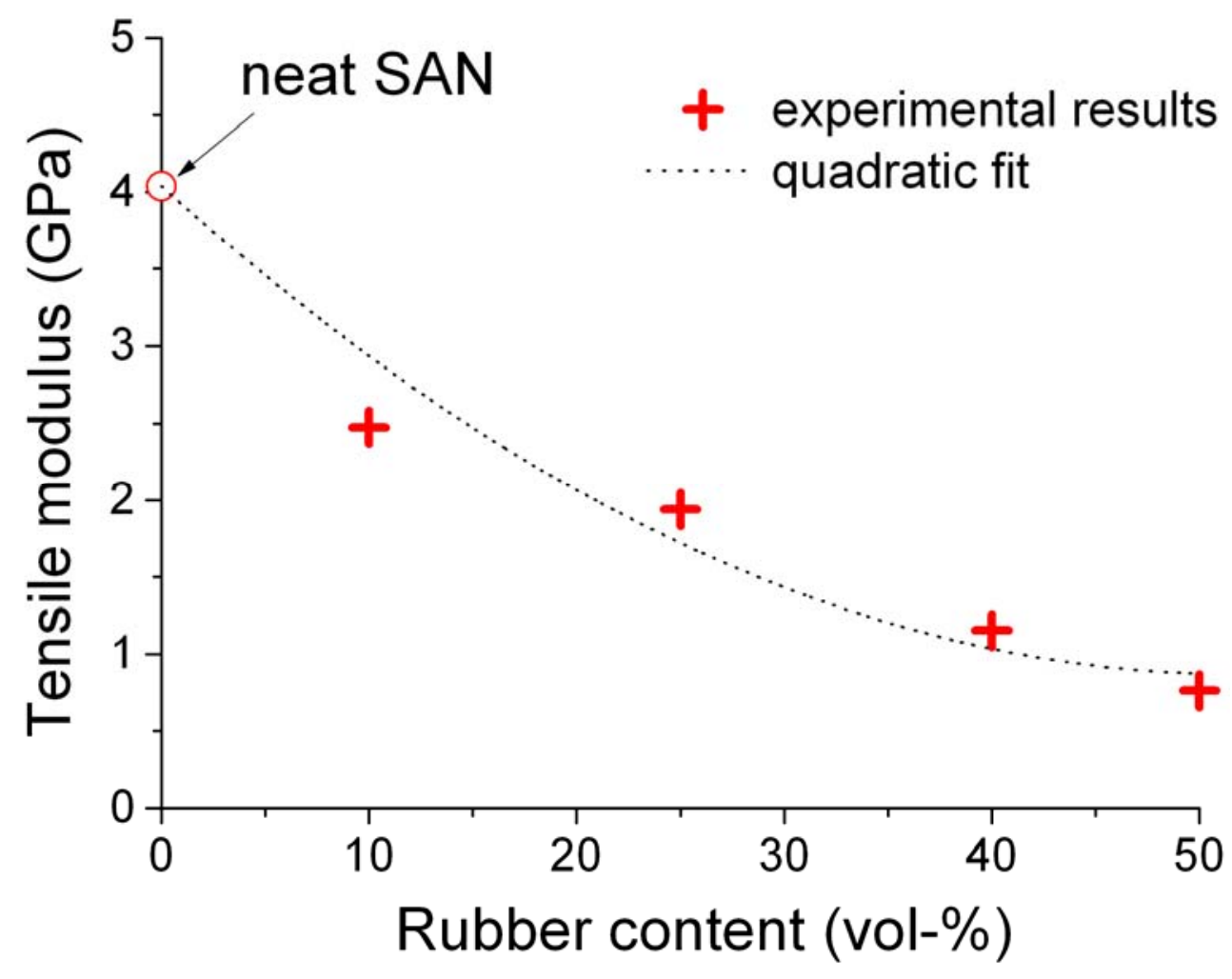

Figure 20. Effect of rubber content on the modulus of SAN. Dotted line: empirical fit (quadratic).

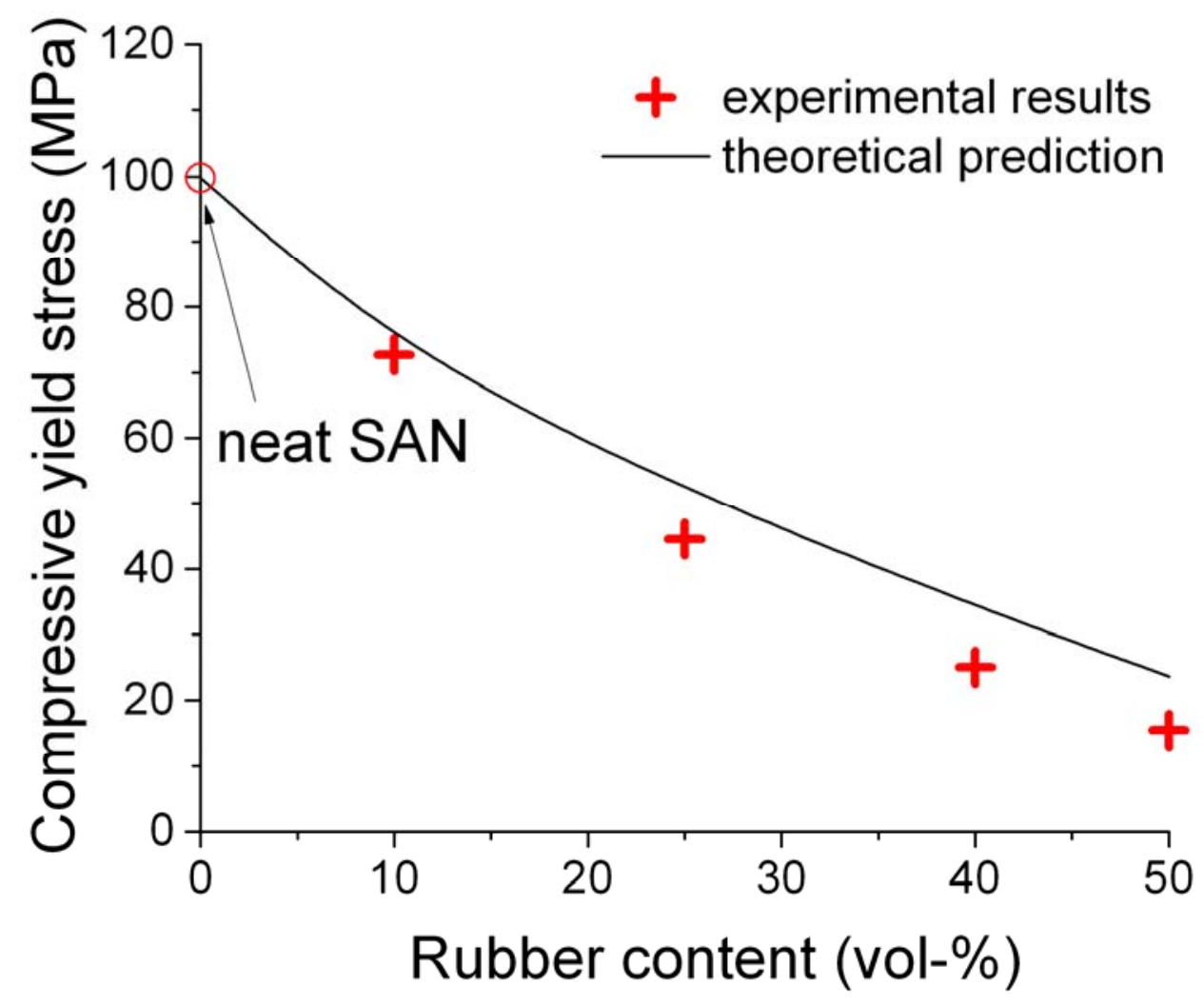

Figure 21. Effect of rubber content on the yield stress of SAN. 
Figure 22 illustrates the correlation between the scratch hardness and the yield stress of SAN-rubber composites. Scratch hardness of SAN decreases drastically with the increasing rubber content. As in the case of toughened epoxy, the same trend observed in the scratch resistance of neat polymers is obeyed also for SAN-rubber composites, whose hardness decreases proportionally to the measured compressive yield stress.

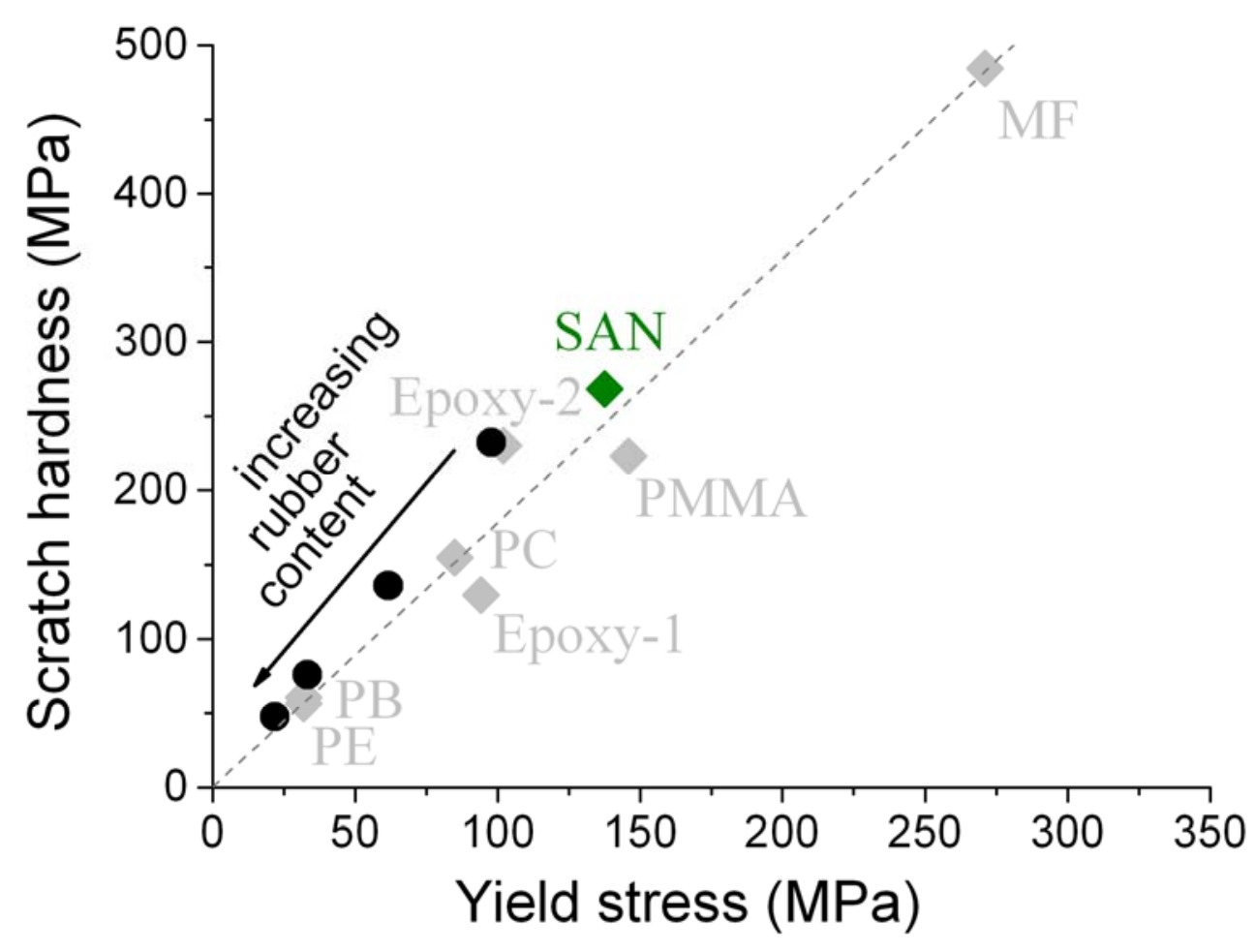

Figure 22. Correlation between scratch hardness and yield stress (at $0.3 \mathrm{~s}^{-1}$ strain rate) of SAN-rubber composites. Greyed data points correspond to data reported in [34].

\section{Discussion}

In general, the addition of fillers modifies the modulus in a way predictable by existing theories, although with a different degree of accuracy in the opposite cases of hard or soft particles; as expected, the overall modulus of the composite material increases or decreases with the addition of particles harder or softer than the matrix, respectively. This symmetry of behaviour seems to be broken when the (compressive) yield stress is considered. While the latter is reduced by the addition of soft fillers as expected, with fillers harder than the matrix the yield stress turned out to be either not affected or only slightly decreasing. This may be related to the degree of adhesion existing between particles and matrix, which can at least partially default at the larger strains.

In addition, a significantly different behaviour was observed for the two matrix polymers with the same (hard) filler: the reinforcing effect of glass spheres on modulus of SAN was in line with theoretical predictions but it was significantly less pronounced for epoxy. Also, there was no clear trend between scratch hardness and filler amount for epoxy, which leads to suspect that particle dispersion and/or adhesion with the matrix was not optimal in the latter case. 
Regarding soft filler composites, a noteworthy different trend in both modulus and yield stress was observed on varying the composition of toughened epoxy, indicative of a phase inversion occurring at a toughener volume content of about $16 \%$. For this amount of added 'filler' a peculiar scratch behaviour was observed, with large oscillations in the recorded penetration depth trace, in contrast with the relatively flat signal recorded at both lower and higher contents of the toughening agent.
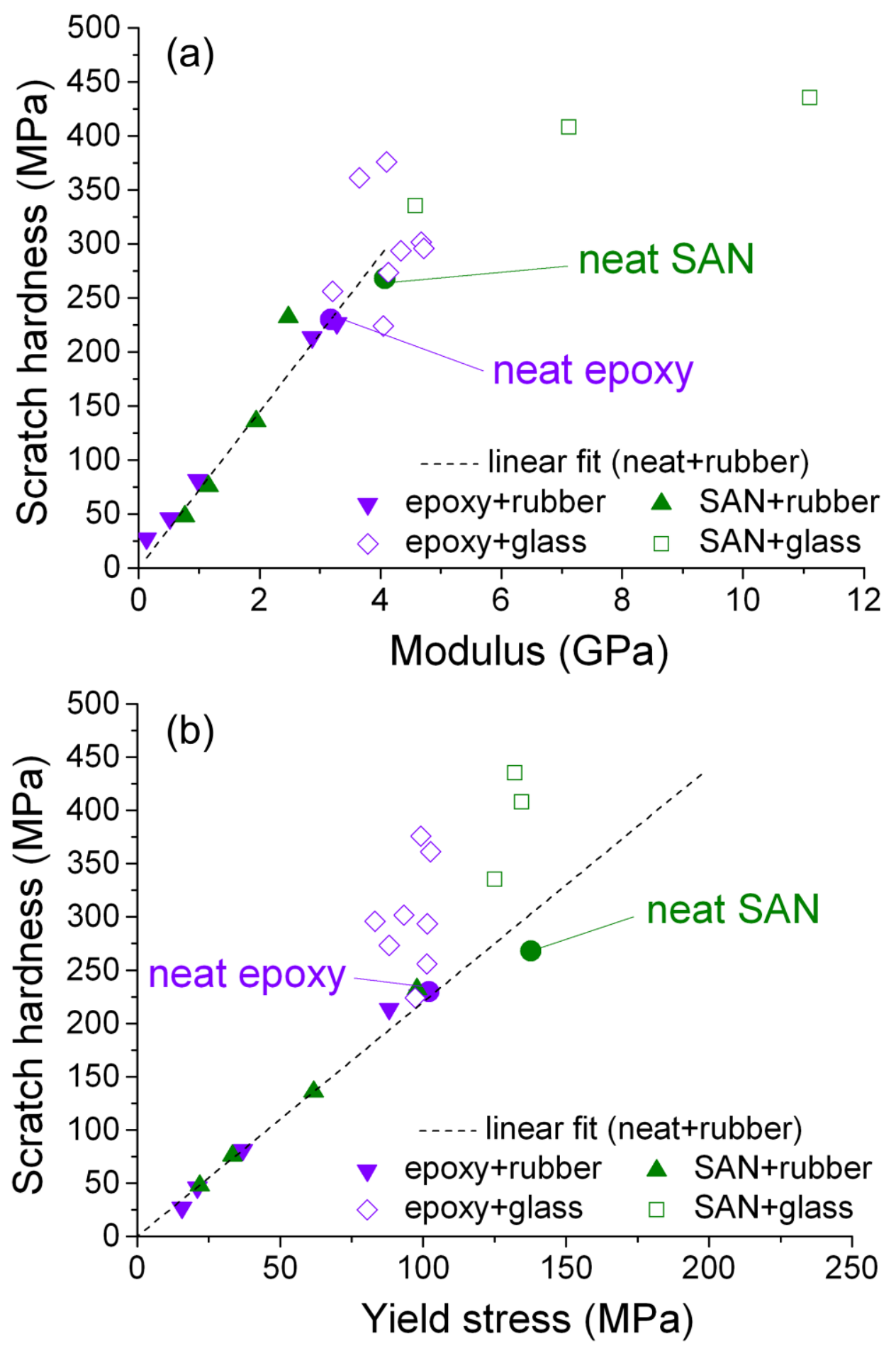

Figure 23. Correlation between scratch hardness and (a) modulus and (b) yield stress of all the materials investigated in the present study. Dashed lines represent a linear fit of data obtained on neat polymers and soft filler composites 
The scratch performance of particulate composites should be viewed in the light of relevant size effects. Scratch is a very local phenomenon with associated characteristic lengths, either instrumental (indenter size) or behavioural (penetration depth). In the present work, the hard particles (glass spheres) had a size comparable with these geometrical quantities and the possible interaction between indenter and each single particle can play an important role. Instead, the dimensions of the soft component domains in the composite samples were significantly smaller and the composite material behaved mechanically like an equivalent, "softer", homogeneous polymer.

It is worth noting that the incorporation of hard fillers resulted in a change of the scratch deformation mode from ductile ploughing to brittle failure, with chip and debris formation. This change brings about a remarkable increase in the scratch visibility which was evaluated simply by visual inspection. A more detailed, quantitative analysis of the loss in optical shine could be implemented using an optical reflectivity method such as the one proposed in [31]. Such an analysis can give very useful information, especially for applications in which the optical quality of components is of paramount importance (e.g. in the automotive sector). In the present work, however, the analysis would be out of scope since the investigation mainly deals with the mechanical aspects of scratch phenomena and, on the other hand, the change in behaviour from neat to glass-filled materials was very evident, as demonstrated by the optical micrographs shown in section 4.

As for scratch hardness, the results are summarized in Figure 23 (a and b). It is evident that with rigid fillers the correlation between scratch hardness and the bulk mechanical properties - specifically the yield stress - does not hold. For SAN-based composites scratch hardness seems to increase regularly with increasing the apparent, overall modulus of the composite, although at a lower rate than previously observed, but with yield stress no correlation exists. For epoxy-based composites no correlation at all is reported with either of the two overall mechanical properties - modulus and yield stress - of the composite. Therefore, for the case of hard fillers it should be concluded that the apparent increase in scratch hardness is related to local interactions between the particles and the indenter.

The behaviour of composites with soft particles falls in line with that previously observed for unfilled polymers. In this case there seems to be no other influence of filler addition on scratch resistance but the effect the particles have on the overall yield stress.

\section{Conclusions}

The effect of the incorporation of different types and amounts of fillers on the scratch behaviour of epoxy- and SAN-based composites was investigated in this study. For the sake of interpreting the results in the light of a model previously assessed for neat polymers, the same composite samples were characterised also in terms of basic mechanical properties, Young's modulus and (compressive) yield stress.

Bulk mechanical properties were compared with predictions from existing theoretical models. A reasonable agreement was found for the tensile modulus, increasing or decreasing with filler content depending on the nature of filler used (hard or soft). A decrease in the yield stress was observed for composites including soft fillers, as expected, while almost no effect was reported in the case of hard fillers.

Hard fillers increased scratch hardness at the price of a larger visibility due to a change from ductile to brittle scratching mode. This variation is not directly related to a change in the composites' mechanical properties. On the other hand, soft fillers diminish the composites' 
scratch hardness consistently with the related reduction in their compressive yield stress. Results highlight the important role played by particle size in relating bulk and surface mechanical properties.

\section{Appendix}

Although for the scratch data analysis only the experimental values of modulus and yield stress were used in this work, a check against values theoretically predicted can be useful for the interpretation of the same scratch results. Relevant theoretical predictions (such as those referred to in Figures 6,11and 21) are briefly recollected in this Appendix.

No account is given here for possible viscoelastic effects. Most theoretical analyses are for elastic response and the results are given in terms of elastic moduli. Extension of the elastic models to viscoelastic materials can be easily accomplished, at least within the linear approximation, by application of the elastic/viscoelastic corresponding principle or the quasi-elastic method. In general, the forms of the composite properties are identical, with the difference that phase complex moduli replace phase elastic moduli [41]. In applying the equations and models it is also customarily assumed that the complex dynamic Poisson's ratio can be replaced by its elastic value. Here it is further assumed that, at the angular frequency used for the experimental determination of the dynamic modulus by DMA, the storage dynamic moduli are comparable to quasi-static or elastic moduli.

\section{A1. Hard particle composites}

\section{A.1.1. Modulus}

As recalled in the text, the theoretical prediction of the elastic moduli of particulate filler in a polymer matrix composite is the subject of a vast literature, using different models and approaches. Mostly the theoretical treatments refer to binary composites and assume perfect adhesion between filler particle and polymer matrix at their interface, which, as noted by Nielsen [36], often results in overestimating the modulus of the composites containing particles harder than the matrix.

Recently, Hsieh et al. [42-43], with a view to sorting out whether a 'nano-effect' does indeed exist in composites containing nano-sized fillers, carefully re-examined the issue. In particular these authors considered the basic Lewis-Nielsen model [44] and the works of McGee and McCullough [45] and Nielsen and Landel [46], allowing for varying degree of adhesion between the matrix polymer and the filler particles. The modulus dependence on filler content predicted for the 'no-slip' case (i.e. very good adhesion between filler and matrix) turned out very similar to the prediction of the widely quoted Halpin-Tsai model [47]; instead, for the 'slip' case (i.e. relatively low adhesion at the filler/matrix interface), the values predicted by the modified Nielsen model turned out much lower.

Following Hsieh et al. [42-43] the modified Nielsen model was applied here to the two hard particle composites considered (epoxy/glass-spheres and SAN/glass-spheres, Figures 6 and 11 respectively), taking

$$
E=\frac{1+\left(k_{E}-1\right) \beta \phi}{1-\mu \beta \phi} E_{m}
$$


for the expression of the Young's modulus of the composite, $E$, as a function of the modulus of the matrix, $E_{m}$, and of the particles, $E_{p}$, and the volume fraction $\phi$ of the particles, with

$$
\beta=\left(\frac{E_{p}}{E_{m}}-1\right) /\left(\frac{E_{p}}{E_{m}}+\left(k_{E}-1\right)\right)
$$

where $k_{E}$ is the generalized Einstein coefficient. Assuming a value of 0.35 for the Poisson's ratio of the polymer matrix, $k_{E}=2.167$ if there is no slippage, or $k_{E}=0.867$ if there is slippage at the matrix-particle interface, as reported in [42-43], and

$$
\mu=1+\frac{(1-\phi)}{\phi_{\max }}\left[\phi_{\max } \phi+\left(1-\phi_{\max }\right)(1-\phi)\right]
$$

is a factor taking into account particle type and packing, with $\phi_{\max }$ being the maximum fraction of particles that can be incorporated in the composite. From [46] $\phi_{\max }=0.632$ for random close-packed, non-agglomerated spheres, as it appears to be the case of both the epoxy/glass-spheres samples and the SAN/glass-spheres samples in the present work. $E_{p}$ was given a value of $70 \mathrm{GPa}$, in both cases.

The predictions of the two models, 'slip' and 'no-slip', for the two systems, epoxy/glass spheres and SAN/glass spheres are displayed as solid lines in Figures 6 and 11, respectively.

\section{A.1.2. Yield stress}

Attempts at predicting the various trends observed experimentally for the yield stress of rigid polymer composites containing more rigid dispersed particles are ill assessed. The uncertainty is increased by the confusion between strength (identified as an ultimate stress) and true yield stress (identified as the threshold to plastic deformations) and by the experimental difficulty in pinpointing this threshold. More fundamentally, it can even be questioned whether an effective overall yield stress, associated to the onset of diffuse plastic deformations, can be defined at all for composites.

No attempt was therefore done here to compare the experimental data of Figures 7 and 12 with any theoretical prediction.

\section{A.2. Soft particle composites}

As noted by McCullogh [38] the behaviour of composites with inclusions more compliant than the matrix should tend toward the limits predicted by upper bound models (analogy to parallel-connected springs). With particles order-of-magnitude softer than the embedding matrix the different theoretical (or semi-empirical) equations almost collapse one onto the other [36-38] but their predictive capacity remains uncertain.

\section{A.2.1. Modulus}

As recognized early by Bucknall [48], with rubber-modified plastics an additional problem arises in attempting to predict the moduli of the particulate composites: the dispersed rubber particles in most rubber-modified plastics are themselves composite in structure, with the rubber embedding some amount of the matrix polymer as small sub-inclusions. The 
distinction between nominal rubber content and effective rubber phase volume (enclosing hard sub-inclusions) is critical and the problem of relating modulus to structure becomes problematic.

No attempt was done here to compare the experimental data of Figure 20 with any theoretical prediction.

\section{A.2.2. Yield stress}

The theoretical prediction of the yield stress in soft particle composites such as rubbertoughened plastics presents the same difficulties, if not more, as the prediction of the modulus. Yet one model, the 'effective area model' proposed by Ishai and Cohen [40], has been used with success by a number of authors, gaining popularity despite its limitations [48]. The model ignores local variations in stress within the matrix and simply calculates an average stress borne by the matrix in the regions where yielding is taking place. According to the model, in a tensile test the stress concentration factor is the ratio of the area of the specimen cross-section to the 'effective area' of the matrix and the latter is calculated on the assumption that the shear bands or crazes responsible for yielding follow a minimum-area path through the matrix, and therefore pass through the centre of each particle they encounter. Assuming that the particles are of spherical shape, the model predicts that the tensile yield stress, $\sigma_{\mathrm{y}}$, of the composite is related to the yield stress of the pure matrix, $\sigma_{\mathrm{ym}}$, and to the volume fraction of particles, $\phi$, by the equation

$$
\sigma_{\mathrm{y}}=\sigma_{\mathrm{ym}}\left(1-1.21 \cdot \phi^{2 / 3}\right)
$$

In this form the model strictly applies to the case of composites containing spherical voids or unbounded particles effectively acting as holes. In ABS polymers (SAN matrix containing SAN-grafted polybutadiene particles) grafting should ensure good adhesion between particles and matrix. However, rubber has a very low shear modulus compared with the glassy polymer matrix and the proportion of hard sub-inclusions, which may raise the shear modulus of the particle, is modest in ABS polymers, so that a rubber particle may effectively act as a hole.

The prediction of this model for the ABS polymer is displayed as a solid line in Fig. 21.

\section{A.3. Systems with phase inversion}

Cases of heterogeneous, two-phase polymer systems showing phase inversion on varying composition are abundantly reported in the literature. Their effective thermo-mechanical properties typically display an S like (or inverted-S like) trend in their dependence on composition, as observed here for both the modulus and the yield stress of the toughened epoxy system (Figures 15 and 16). Physical arguments for the observed behaviour are well assessed but theoretical models are scanty or just semi-empirical [36, 49].

No attempt was therefore done here to fit the experimental data points of Figures 15 and 16 to theoretical or semi-empirical relationships. 


\section{Acknowledgements}

Most of the research work reported in the present paper is part of Pinar Kurkcu's PhD Thesis in Materials Engineering, Politecnico di Milano (2011). The authors wish to thank Mr. Oscar Bressan for specimen preparation.

\section{References}

[1] J.S.S. Wong, H.J. Sue, Scratch Behaviour of Polymers, in: Encyclopedia of Polymer Science and Technology, 3rd Ed., Vol.11, John Wiley and Sons, 2004.

[2] B.J. Briscoe, S.K. Sinha, Scratch resistance and localized damage characteristics of polymer surfaces-a review, Materialwiss. Werkstofftech. 34 (2003) 989-1002.

[3] V. Jardret, Understanding and quantification of elastic and plastic deformation during a scratch test, Wear 218 (1998) 8-14.

[4] H. Pelletier, A.L. Durier, C. Gauthier, R. Schirrer, Viscoleastic and elastic-plastic behaviours of amorphous polymeric surfaces during scratch, Tribol. Int. 41 (2008) 975-984.

[5] B.J. Briscoe, P.D. Evans, E. Pelillo, S.K. Sinha, Scratching maps for polymers, Wear 200 (1996) 137-147.

[6] L.C.A. Van Breemen, L.E. Govaert, H.E.H. Meijer, Scratching polycarbonate: A quantitative model, Wear 274-275 (2012) 238-247.

[7] N.L. Surampadi, T.C. Pesacreta, R.D.K. Misra, The determining role of scratch indenter radius on surface deformation of high density polyethylene and calcium carbonate-reinforced composite, Mater. Sci. Eng. A 456 (2007) 218-229.

[8] A. Krupicka, M. Johansson, A. Hult, Use and interpretation of scratch tests on ductile polymer coatings, Prog. Org. Coat. 46 (2003) 32-48.

[9] J.S.S. Wong, H.J. Sue, K.Y. Zeng, R.K.Y. Li, Y.W. Mai, Scratch damage of polymers in nanoscale, Acta Mater. 52 (2004) 431-443.

[10] M.Wong, G.T. Lim, A. Moyse, J.N. Reddy, H.J. Sue, A new test methodology for evaluating scratch resistance of polymers, Wear 256 (2004) 1214-1227.

[11] S.L. Zhang, J.C.M. Li, Slip process of stick-slip motion in the scratching of a polymer, Mater. Sci. Eng. A 344 (2003) 182-189.

[12] V. Jardret, P. Morel, Viscoelastic effects on the scratch resistance of polymers: relationship between mechanical properties and scratch properties at various temperatures, Prog. Org. Coat. 48 (2003) 332-331.

[13] C. Gauthier, R. Schirrer, Time and temperature dependence of the scratch properties of poly(methylmethacrylate) surfaces, J. Mater. Sci. 35 (2000) 2121-2130.

[14] Y. Hara, T. Mori, T. Fujitani, Relationship between viscoelasticity and scratch morphology of coating films, Prog. Org. Coat. 40 (2000) 39-47.

[15] C. Xiang, H.J. Sue, J. Chu, B. Coleman, Scratch behaviour and material property relationship in polymers, J. Polym. Sci., Part B: Polym. Phys.39 (2001) 47-59.

[16] J. Rodriguez, A. Rico, V. Soria, Tribological properties of commercial optical disks estimated from nanoindentation and scratch techniques, Wear 263 (2007) 1545-1550.

[17] R.L. Browning, G.T. Lim, A. Moyse, H.J. Sue, H. Chen, J.D. Earls, Quantitative evaluation of scratch resistance of polymeric coatings based on standardized progressive load scratch test, Surf. Coat. Technol.201 (2006) 2970-2976. 
[18] R.S. Hadal, R.D.K. Misra, Scratch deformation behaviour of thermoplastic materials with significant differences in ductility, Mater. Sci. Eng. A 398 (2005) 252-261.

[19] E. Moghbelli, R.L. Browning, W.J. Boo, S.F. Hahn, L.J.E. Feick, H.J. Sue, Effects of molecular weight and thermal history on scratch behaviour of polypropylene thin sheets, Tribol. Int. 41 (2008) 425-433.

[20] B.J. Briscoe, P.D. Evans, S.K. Biswass ad S.K. Sinha, The hardness of poly(methylmethacrylate), Tribol. Int. 29 (1996) 93-104.

[21] W. Brostow, P.E. Cassidy, J. Macossay, D. Pietkiewicz, S. Venumbaka, Connection of surface tension to multiple tribological properties in epoxy+fuoropolymer systems, Polym. Int. 52 (2003) 1498-1505.

[22] A. Kopczynska, G.W. Ehrenstein, Polymeric surfaces and their true surface tension in solids and melts, J. Mater. Ed. 29 (2007) 325-340.

[23] Briscoe, B.J. and Sinha, S.K., Tribological Applications of Polymers and their Composites: Past, Present and Future Prospects in Tribology of Polymeric Nanocomposites, Tribol. Interface Eng. Ser. 55 (2008) 1-14.

[24] R.R. Thridandapani, A. Mudaliar, Q. Yuan, R.D.K. Misra, Near surface deformation associated with the scratch in polypropylene-clay nanocomposite: A microscopic study, Mater. Sci. Eng. A 418 (2006) 292-302.

[25] J. Chu, C. Xiang, H.J. Sue, R. Damon Hollis, Scratch Resistance of Mineral-Filled Polypropylene Materials, Polym. Eng. Sci. 40, (2000) 944-955.

[26] R. Hadal, A. Dasari, J. Rohrmann, R.D.K. Misra, Susceptibility to scratch surface damage of wollastonite- and talc-containing polypropylene micrometric composites, Mater. Sci. Eng. A 380 (2004) 326-339.

[27] R.D.K. Misra, R. Hadal, S.J. Duncan, Surface damage behavior during scratch deformation of mineral reinforced polymer composites, Acta Mater. 52 (2004) 4363-4376.

[28] R.D.K. Misra, H. Nathani, A. Dasari, S.D. Wanjale, J.P. Jog, The determining role of clay particles on mechanically induced surface damage and associated stress whitening in polybutene-clay nanocomposites, Mater. Sci. Eng. A 386 (2004) 175-185.

[29] E. Moghbelli, L. Sun, H. Jiang, W.J. Boo, H.J. Sue, Scratch Behavior of Epoxy Nanocomposites Containing a-Zirconium Phosphate and Core-Shell Rubber Particles, Polym. Eng. Sci. 49 (2009) 483490.

[30] C. Xiang, H.J. Sue, J. Chu, K. Masuda, Roles of Additives in Scratch Resistance of High Crystallinity Polypropylene Copolymers, Polym. Eng. Sci. 41 (2001) 23-31.

[31] B.J. Briscoe, E. Pelillo, S.K. Sinha, Characterisation of the scratch deformation mechanisms for poly(methylmethacrylate) using surface optical reflectivity, Polym. Int. 43 (1997) 359-367.

[32] S.K. Sinha, T. Song, X. Wan, Y. Tong, Scratch and normal hardness characteristics of polyamide 6/nano-clay composite, Wear 266 (2009) 814-821.

[33] K.L. Johnson, The correlation of indentation experiments, J. Mech. Phys. Solids 18 (1970) 115126.

[34] P. Kurkcu, L. Andena, A. Pavan. An experimental investigation of the scratch behaviour of polymers: 1. influence of rate-dependent bulk mechanical properties. Wear 290-291 (2012) 86-93.

[35] S.K. Garg, V. Svalbonas, G.A.Gurtman, Analysis of Structural Composite Materials, Marcel Dekker, Inc., New York, 1973.

[36] L.E. Nielsen, Mechanical Properties of Polymers and Composites, Vol. 2, first ed., Marcel Dekker, Inc., New York, 1974. 
[37] T.S. Chow, The effect of particle shape on the mechanical properties of filled polymers, J. Mater. Sci. 15 (1980) 1873-1888.

[38] R.L. Mc Cullough, Micro-models for composite materials - Particulate and discontinuous fiber composites, Ch. 2.4 in Delaware Composites Design Encyclopedia, Vol. 2 (1990) 93.

[39] S.-Y. Fu, X.-Q. Feng, B. Lauke, Y.W. Mai, Effects of particle size, particle/matrix interface adhesion and particle loading on mechanical properties of particulate-polymer composites, Composites, B, 39 (2008) 933-961.

[40] O. Ishai, L.J. Cohen, Effect of Fillers and Voids on Compressive Yield of Epoxy Composites. J. Compos. Mat. 2, (1968) 302-315.

[41] R.A. Dickie, The viscoelastic properties of particulate polymeric composites, Ch. 3 in Polymer Engineering Composites, M.O.W. Richardson, ed., Appl. Sci. Publ. Ltd, London, 1977.

[42] T.H. Hsieh, A.J. Kinloch, K. Masania, J. Sohn Lee, A.C. Taylor, S. Sprenger, The toughness of epoxy polymers and fibre composites modified with rubber microparticles and silica nanoparticles, J. Mater. Sci., 45 (2010) 1193-1210.

[43] T.H. Hsieh, A.J. Kinloch, K. Masania, A.C. Taylor, S. Sprenger, The mechanisms and mechanics of the toughening of epoxy polymers modified with silica nanoparticles, Polymer, 51 (2010) 62846294.

[44] T.B. Lewis, L.E. Nielsen, Dynamic mechanical properties of particulate-filled composites, J. Appl. Polym. Sci., 14(6) (1970) 1449-1471.

[45] S. McGee, R.L. McCullough, Combining rules for predicting the thermoelastic properties of particulate filled polymers, polymers, polyblends, and foams, Polym. Compos. 2 (1981) 149-161.

[46] L.E. Nielsen, R.F. Landel, Mechanical properties of polymers and composites, Marcel Dekker, New York, $2^{\text {nd }}$ ed. (1994).

[47] J.C. Halpin, J.L. Kardos, The Halpin-Tsai equations: a review, Polym. Eng. Sci. 16 (1976) 344352.

[48] C.B. Bucknall, Toughened Plastics, Applied Science, London (1977).

[49] L.H. Sperling, Microphase structure, in Encyclopedia of Polymer Science and Engineering, $2^{\text {nd }}$ ed., H.F. Mark, N.M. Bikales, C.G. Overberger, G. Menges, eds., Wiley, New York (1987), Vol.9, 760-788. 


\section{Figure Captions}

Figure 1. 50x magnification optical micrographs of epoxy-glass composites having different sphere diameter and volume content: a) $100 \mu \mathrm{m}, 10$ vol- $\%$; b) $100 \mu \mathrm{m}, 30$ vol- $\%$; c) $25 \mu \mathrm{m}$, 10 vol- $\%$; d) $25 \mu \mathrm{m}, 30$ vol- $\%$.

Figure 2. Correlation between scratch hardness and yield stress in neat polymers [32]. The material labelled as "Epoxy-2" is the same epoxy resin investigated in the present study.

Figure 3. Above: examples of penetration depth, residual depth and acoustic emission records plotted as a function of scratch length. Below: examples of optical micrographs of top views of a scratch groove; (a) neat epoxy and (b) epoxy composite with 40 vol-\%. of glass spheres (diameter $=100 \mu \mathrm{m})$.

Figure 4. Penetration depth, residual depth and acoustic emission records plotted as a function of scratch length of epoxy-based composites filled with $100 \mu \mathrm{m}$ glass spheres in varying amounts: (a) 10 vol-\%. (b) 20 vol-\%. (c) 30 vol-\%. (d) 40 vol- $\%$.

[Note - Fig. 4(d) identical to Fig. 3(b) above.]

Figure 5. Penetration depth, residual depth and acoustic emission records plotted as a function of scratch length of epoxy-based composites filled with glass spheres having a diameter of $25 \mu \mathrm{m}$ in varying amounts: (a) 10 vol-\%. (b) 20 vol-\%. (c) 30 vol- $\%$. (d) 40 vol- $\%$.

Figure 6. Effect of incorporation of glass spheres on the storage tensile modulus of epoxy-based composites (frequency $=1 \mathrm{~Hz}, d=$ filler diameter). Dashed line: empirical linear fit, continuous lines: theoretical predictions according to the "modified" Nielsen model (see Appendix).

Figure 7. Effect of incorporation of glass spheres on the yield stress in compression of epoxy based composites (strain rate: $10^{-4} \mathrm{~s}^{-1}, d=$ glass sphere diameter).

Figure 8. Correlation between scratch hardness and yield stress of epoxy-glass sphere composites. Greyed points correspond to data for neat polymers reported in [32].

Figure 9. Above: examples of penetration depth, residual depth and acoustic emission records and, below: examples of optical micrographs of top view of a scratch groove, for (a) neat SAN and (b) SAN composite containing 40 vol- $\%$ of glass spheres (diameter $=25 \mu \mathrm{m}$ ).

Figure 10. Penetration depth, residual depth and acoustic emission records of SAN-based composites filled with glass spheres having a diameter of $25 \mu \mathrm{m}$ in amounts of: (a) $10 \mathrm{vol}-\%$, (b) 25 vol- $\%$ and (c) 40 vol- $\%$.

Figure 11. Effect of incorporation of glass spheres on the storage modulus of SAN at $1 \mathrm{~Hz}$. Dotted line: empirical fit (quadratic). Solid lines: theoretical predictions according to the "modified" Nielsen model (see Appendix).

Figure 12. Effect of incorporation of glass spheres on the yield stress of SAN (strain rate: $\left.10^{-4} \mathrm{~s}^{-1}\right)$. Dashed line: empirical fit (linear).

Figure 13. Correlation between scratch hardness and yield stress (at $0.3 \mathrm{~s}^{-1}$ strain rate) of SAN / glass sphere composites. Greyed points correspond to data for neat polymers reported in [32]. 
Figure 14. Above: examples of penetration depth, residual depth and acoustic emission records of neat (a) and toughened epoxy containing 5 vol- $\%$ (b), 10 vol- $\%$ (c), 20 vol- $\%$ (d), 30 vol-\% (e) and 40 vol-\% (f) of toughening agent. Below: examples of optical micrographs of top view of scratch grooves for toughened epoxies containing 5 vol- $\%$ (b) and 20 vol- $\%$ (d) of toughening agent.

Figure 15. Effect of toughening agent content on the modulus of epoxy composites. Dashed line: empirical fitting (Equation 1 with $x_{0}=16.1 \pm 0.9 ; p=4.4 \pm 0.9$ ).

Figure 16. Effect of toughening agent content on the yield stress of epoxy composites. Dashed line: empirical fit (Equation 1 with $x_{0}=15.4 \pm 0.4 ; p=3.9 \pm 0.3$ ).

Figure 17. Correlation between scratch hardness and yield stress (at $0.3 \mathrm{~s}^{-1}$ strain rate) of toughened epoxy. Greyed data points correspond to data reported in [32].

Figure 18. Effect of toughening agent content on the normalized (with respect to the neat polymer value) elastic modulus $E$, yield stress $\sigma_{\mathrm{y}}$ and scratch hardness $H_{\mathrm{S}}$ of toughened epoxy. Dashed line: empirical fit (Equation 1 with $x_{0}=16.8 \pm 1.0 ; p=3.0 \pm 0.5$ ).

Figure 19. Penetration depth, residual depth and acoustic emission records of neat (a) and rubber-toughened SAN including 10 vol-\%. (b), 25 vol-\%. (c) and 50 vol-\%. (d) of rubber.

Figure 20. Effect of rubber content on the modulus of SAN. Dotted line: empirical fit (quadratic).

Figure 21. Effect of rubber content on the yield stress of SAN.

Figure 22. Correlation between scratch hardness and yield stress (at $0.3 \mathrm{~s}^{-1}$ strain rate) of SAN-rubber composites. Greyed data points correspond to data reported in [32].

Figure 23. Correlation between scratch hardness and (a) modulus and (b) yield stress of all the materials investigated in the present study. Dashed lines represent a linear fit of data obtained on neat polymers and soft filler composites 